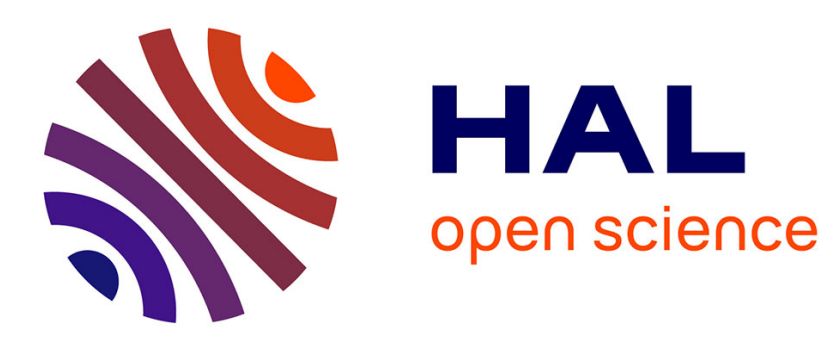

\title{
Analytical preconditioners for Neumann elastodynamic Boundary Element Methods
}

\author{
Stéphanie Chaillat, Marion Darbas, Frédérique Le Louër
}

\section{To cite this version:}

Stéphanie Chaillat, Marion Darbas, Frédérique Le Louër. Analytical preconditioners for Neumann elastodynamic Boundary Element Methods. SN Partial Differential Equations and Applications, 2021, 2 (22), 10.1007/s42985-021-00075-x . hal-02512652v2

\section{HAL Id: hal-02512652 \\ https://hal.science/hal-02512652v2}

Submitted on 6 Oct 2021

HAL is a multi-disciplinary open access archive for the deposit and dissemination of scientific research documents, whether they are published or not. The documents may come from teaching and research institutions in France or abroad, or from public or private research centers.
L'archive ouverte pluridisciplinaire HAL, est destinée au dépôt et à la diffusion de documents scientifiques de niveau recherche, publiés ou non, émanant des établissements d'enseignement et de recherche français ou étrangers, des laboratoires publics ou privés. 


\title{
Analytical preconditioners for Neumann elastodynamic boundary element methods
}

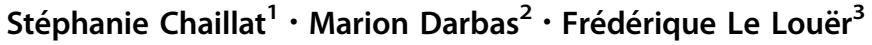

Received: 30 March 2020 / Accepted: 3 February 2021 / Published online: 2 March 2021

(C) The Author(s), under exclusive licence to Springer Nature Switzerland AG part of Springer Nature 2021

\begin{abstract}
Recent works in the boundary element method (BEM) community have been devoted to the derivation of fast techniques to perform the matrix-vector product needed in the iterative solver. Fast BEMs are now very mature. However, it has been shown that the number of iterations can significantly hinder the overall efficiency of fast BEMs. The derivation of robust preconditioners is now inevitable to increase the size of the problems that can be considered. Analytical preconditioners offer a very interesting strategy by improving the spectral properties of the boundary integral equations ahead of discretization. The main contribution of this paper is to propose new analytical preconditioners to treat Neumann exterior scattering problems in 2D and 3D elasticity. These preconditioners are local approximations of the adjoint Neumann-toDirichlet map. We propose three approximations with different orders. The resulting boundary integral equations are preconditioned Combined Field Integral Equations (CFIEs). An analytical spectral study confirms the expected behavior of the preconditioners, i.e., a better eigenvalue clustering especially in the elliptic part contrary to the standard CFIE of the firstkind. We provide various 2D numerical illustrations of the efficiency of the method for smooth and non smooth geometries. In particular, the number of iterations is shown to be independent of the density of discretization points per wavelength which is not the case for the standard CFIE. In addition, it is less sensitive to the frequency.
\end{abstract}

Keywords Scattering - Time-harmonic elastic waves - Boundary element method · Analytical preconditioner · Approximate local Neumann-to-Dirichlet map · Cavity

Mathematics subject classification $65 \mathrm{~N} 38 \cdot 65 \mathrm{~F} 08 \cdot 45 \mathrm{~B} 05 \cdot 47 \mathrm{G} 30$

\section{Introduction}

The development of numerical methods for solving highly oscillatory elastic scattering problems is of great interest in medical or industrial applications (for example elastrography imaging, seismology, geophysical exploration or non-destructive testing). This paper

This article is part of the topical collection "Waves 2019_invited papers" edited by Manfred Kaltenbacher and Markus Melenk. 
considers the solution of scattering problems of time-harmonic elastic waves by a two- or three-dimensional bounded obstacle with a Neumann boundary condition. Various numerical approaches exist to deal with exterior boundary-value problems [60]. We mention spectral methods [24, 44, 50], Finite Element Methods [9, 41] or finite difference methods $[39,57]$. For the class of volume methods, the unbounded computational domain must be truncated using an artificial boundary at a finite distance on which a boundary condition is imposed. Many possibilities are proposed in the literature: nonreflecting boundary conditions [37, 38] such as Absorbing Boundary Conditions (ABCs) [18, 29, 34] or perfectly matched layers (PMLs) $[8,26,40]$. The method of boundary integral equations (BIEs) is another classical tool for solving scattering problems of time-harmonic waves in unbounded, homogeneous and isotropic media (see e.g. [10, 42, 48, 49]). The main advantage is to formulate the exterior boundary value-problem as an integral equation on the boundary of the scatterer. Only the surface of the obstacle is thus needed to be meshed in contrast to volume methods. And the dimensionality of the problem is reduced by one.

However, the discretization matrix of a boundary integral operator is dense. Furthermore, in order to capture the oscillatory phenomenon, one has to fix typically about ten discretization points per wavelength per dimension. The solution of these large and fullypopulated complex linear systems is handled by iterative solvers, namely GMRES [61]. The standard Boundary Element Method (BEM) results in high computational costs in terms of computational time $\left(\mathcal{O}\left(N^{2}\right)\right.$ per iteration) and memory requirements $\left(\mathcal{O}\left(N^{2}\right)\right)$, where $N$ denotes the number of degrees of freedom (DOFs) of the BEM model. A number of algorithms have been introduced to evaluate matrix-vector products in a fast way, when the matrix is obtained by the discretization of an integral operator. The Fast Multipole accelerated Boundary Element Method (FM-BEM) is one of the efficient methods. The method has been introduced by Rokhlin [56] and extended to various domains included 3D elastodynamics $[19,20]$. A different kind of compression can be obtained by applying the adaptive cross approximation (ACA) algorithm and hierarchical matrices [7].

In addition, the spectral properties of the most stable integral equation formulations, the Combined Field Integral Equations (CFIEs), are usually not well-suited for Krylov-subspace iterative solvers such as GMRES. The cavity problem is particularly challenging. For such a boundary condition, the standard CFIE is a boundary integral equation of the first kind. It involves the Neumann boundary trace of the double-layer potential which is a pseudodifferential operator of order 1. Consequently, the operator discretized in the CFIE has an unbounded sequence of eigenvalues, and the condition number of the matrix behaves like $\mathcal{O}(1 / h)$ in the standard basis, where $h$ is the mesh size. We will see that this drawback of the CFIE is exacerbated at high frequencies. In this paper, we focus on the construction of new and well-conditioned BIEs which are more robust than the standard CFIE.

Specifically, two families of preconditioners exist. We can cite algebraic preconditioning approaches such as incomplete LU, SParse Approximate Inverse [16, 17], multigrid methods [15], nested GMRES algorithm [23, 45] which have been applied to electromagnetic or elastodynamic fast BEMs. However, since algebraic preconditioners retain only a small contribution of the system matrix, they do not contain enough information on the underlying continuous operator. This approach is effective but shows only moderate efficiency for high frequency problems. Analytical preconditioners offer a very interesting alternative. They act ahead from the discretization. This preconditioning technique based on boundary integral operators of opposite orders, also known as Calderón preconditioning, has been introduced by Steinbach and Wendland [58] in electromagnetism. Since then, several works have been devoted to the derivation of Fredholm boundary integral 
equations of the second kind for both acoustic and electromagnetic scattering problems by closed surfaces (e.g. [1, 4, 5, 11-13, 25, 31, 35, 36, 51, 52, 55, 59]) or open surfaces (e.g. $[3,14,27,28])$. Among them, approximations of the Dirichlet-to-Neumann map (respectively the Neumann-to-Dirichlet map) naturally define robust analytical preconditioners when considering Dirichlet (respectively Neumann) boundary value-problems. They are introduced as regularizing operators in the integral representation of the scattered field and improve the spectral properties of the resulting boundary integral equations. A pseudo inverse of the principal classical symbol of the single layer boundary integral operator-or equivalently the principal classical symbol of the Neumann trace of the double layer boundary integral operator-is used to approach the Dirichlet-to-Neumann map and its adjoint operator [5, 6, 30] in the framework of the On-Surface Radiation Condition (OSRC) methods (e.g. [2, 43, 47]). In acoustics, the resulting preconditioner is expressed analytically by a simple square-root of the form $i \kappa\left(\mathrm{I}+\Delta_{\Gamma} / \kappa^{2}\right)^{1 / 2}$ where $\kappa$ is the wavenumber and $\Delta_{\Gamma}$ the Laplace-Beltrami operator. Using the same techniques of pseudodifferential calculus, recent works have proposed analytical preconditioners for Dirichlet elastic BEMs [32]. Some difficulties inherent to elasticity have to be overcome. For the elasticity case, the double layer boundary integral operator and its adjoint are not compact even for sufficiently smooth boundaries. This implies, according to Calderón's identities, that regularizing the standard BIEs via a pseudo inverse of the single layer boundary integral operator is not sufficient to obtain well-conditioned boundary integral equations. The principal part of the double layer boundary integral operator has also to be taken into account in the preconditioner to regularize the single layer boundary integral operator. It is not an easy task to obtain the expressions of the principal parts of each elementary boundary integral operator. To this end, a modified potential theory is applied and the tangential Günter derivative plays an important role. The approximations of the Dirichletto-Neumann map are expressed in terms of surface differential operators, square-root operators and their inverses. These preconditioners can easily be combined with fast methods such as FMM [31], and yield a very fast convergence of the GMRES solver and in particular with a number of iterations independent of the frequency and the mesh density.

In this paper, we construct analytical preconditioners for the iterative solution of cavity problems. To the best of our knowledge, this is the first contribution in this sense in elastodynamics. Contrary to the acoustic and electromagnetic cases, the definition of the Neumann-to-Dirichlet preconditioner as the inverse of the Dirichlet-to-Neumann preconditioner is not sufficient to cluster all the eigenvalues around the unit number in the complex plane. The approximations of different orders of the adjoint Neumann-toDirichlet map, that we propose to apply as regularizing operators, are derived using strategies developed in [32]. However, we cannot use the modified potential theory and the help of the tangential Günter derivative to overcome the non-compactness of the doublelayer boundary integral operator.

The paper is organized as follows: in Sect. 2, we introduce the problem setting. We present the Combined Field Integral Equation (CFIE) formulations that are numerically investigated in this paper. In Sect. 3, we describe the different approximate adjoint Neumann-to-Dirichlet maps and the corresponding CFIEs in the two- and three-dimensional cases. Section 4 is devoted to analytical investigations of the spectral properties of the standard and preconditioned operators in the particular case of the elastic sphere. Furthermore, we study the effect of both the number of spherical harmonics and the frequency increase on the condition number. In Sect. 5, we provide some elements of the discretization and implementation. We also give various numerical illustrations of the 
efficiency of the method for several 2D geometries. We address numerical investigation of the eigenvalues of the classical and preconditioned CFIEs. Finally, we draw concluding remarks, and we discuss possible research lines in Sect. 6.

\section{The Navier exterior problem and standard boundary integral equations}

\subsection{The Navier exterior problem}

We consider an elastic cavity represented by a bounded domain $\Omega^{-}$in $\mathbb{R}^{d}, d=2,3$, with a closed boundary $\Gamma:=\partial \Omega^{-}$of class $\mathscr{C}^{2}$ at least. Let $\Omega^{+}$denote the exterior domain $\mathbb{R}^{d} \backslash \overline{\Omega^{-}}$ and $\boldsymbol{n}$ the outer unit normal vector to the boundary $\Gamma$. The Lamé parameters $\mu$ and $\lambda$ and the density $\rho$ are positive constants. The propagation of time-harmonic waves in an isotropic and homogeneous elastic medium is governed by the Navier equation [49, Eq. (12.5) page 55]

$$
\mu \Delta \boldsymbol{u}+(\lambda+\mu) \nabla \operatorname{div} \boldsymbol{u}+\rho \omega^{2} \boldsymbol{u}=0,
$$

where $\omega>0$ denotes the angular frequency. The displacement field $\boldsymbol{u}$ is decomposed into a longitudinal field $\boldsymbol{u}_{p}$ (compressional part) with vanishing curl and a transverse divergencefree field $\boldsymbol{u}_{s}$, both solutions to the Helmholtz equation with respective wavenumbers $\kappa_{p}^{2}=$ $\rho \omega^{2}(\lambda+2 \mu)^{-1}$ and $\kappa_{s}^{2}=\rho \omega^{2} \mu^{-1}$. The Neumann trace, defined by $\boldsymbol{t}_{\mid \Gamma}:=\boldsymbol{T} \boldsymbol{u}$, is given by the traction operator

$$
\boldsymbol{T}=2 \mu \frac{\partial}{\partial \boldsymbol{n}}+\lambda \boldsymbol{n} \operatorname{div}+\mu \boldsymbol{n} \times \text { curl. }
$$

The two-dimensional traction is obtained by setting $\boldsymbol{u}=\left(u_{1}, u_{2}, 0\right)$ and $\boldsymbol{n}=\left(n_{1}, n_{2}, 0\right)$ in the above definition.

The elastic cavity problem is formulated as follows : Given an incident displacement wave $\boldsymbol{u}^{i n c}$ which is assumed to solve the Navier equation in the absence of any scatterer, find the scattered field $\boldsymbol{u}$ solution to the Navier equation (1a) in $\Omega^{+}$which satisfies the Neumann boundary condition

$$
\boldsymbol{t}_{\mid \Gamma}=-\boldsymbol{t}_{\mid \Gamma}^{i n c} \quad \text { on } \Gamma
$$

where we have set $\boldsymbol{t}_{\mid \Gamma}^{i n c}=\boldsymbol{T} \boldsymbol{u}^{i n c}$. In addition, the behavior of the scattered displacement field $\boldsymbol{u}$ at infinity is described by the Kupradze radiation conditions [49, Eqs (2.6)-(2.9) page 126]

$$
\lim _{r \rightarrow \infty} r^{(d-1) / 2}\left(\frac{\partial \boldsymbol{u}_{p}}{\partial r}-i \kappa_{p} \boldsymbol{u}_{p}\right)=0, \quad \lim _{r \rightarrow \infty} r^{(d-1) / 2}\left(\frac{\partial \boldsymbol{u}_{s}}{\partial r}-i \kappa_{s} \boldsymbol{u}_{s}\right)=0, \quad r=|\boldsymbol{x}|,
$$

uniformly in all directions.

We denote by $H_{l o c}^{s}\left(\overline{\Omega^{+}}\right)$and $H^{s}(\Gamma)$ the standard (local in the case of the exterior domain) complex valued, Hilbert-Sobolev spaces of order $s \in \mathbb{R}$ defined on $\overline{\Omega^{+}}$and $\Gamma$ respectively (with the convention $H^{0}=L^{2}$ ). Spaces of vector functions will be denoted by boldface letters, thus $\boldsymbol{H}^{s}=\left(H^{s}\right)^{d}$. We set $\boldsymbol{\Delta}^{*} \boldsymbol{u}:=\mu \boldsymbol{\Delta} \boldsymbol{u}+(\lambda+\mu) \boldsymbol{\nabla} \operatorname{div} \boldsymbol{u}$. The radiating solution to (1a)-(1b)-(1c) belongs to the space 


$$
\boldsymbol{H}_{+}^{1}\left(\boldsymbol{\Delta}^{*}\right):=\boldsymbol{H}_{l o c}^{1}\left(\Omega^{+}, \boldsymbol{\Delta}^{*}\right):=\left\{\boldsymbol{u} \in \boldsymbol{H}_{l o c}^{1}\left(\overline{\Omega^{+}}\right): \boldsymbol{\Delta}^{*} \boldsymbol{u} \in \boldsymbol{L}_{l o c}^{2}\left(\overline{\Omega^{+}}\right)\right\} .
$$

For existence and uniqueness results, we refer to Kupradze [48, 49].

\subsection{Potential theory and integral representation}

The first main difficulty arising in the numerical solution to the exterior boundary valueproblem (1a)-(1b)-(1c) is related to the unbounded computational domain $\Omega^{+}$. Methods based on the discretization of boundary integral equations are one possibility to overcome this issue. For any positive real number $\kappa$, let

$$
G(\kappa, x-y)= \begin{cases}\frac{i}{4} H_{0}^{(1)}(\kappa|x-y|), & \text { if } d=2, \\ \frac{e^{i \kappa \mid x-y}}{4 \pi|\boldsymbol{x}-\boldsymbol{y}|}, & \text { if } d=3\end{cases}
$$

be the fundamental solution of the Helmholtz equation $\Delta v+\kappa^{2} v=0$. The fundamental solution of the Navier equation is written

$$
\Phi(\boldsymbol{x}, \boldsymbol{y})=\frac{1}{\mu}\left[G\left(\kappa_{s},|\boldsymbol{x}-\boldsymbol{y}|\right) \cdot \mathrm{I}_{\mathbb{R}^{d}}+\frac{1}{\kappa_{s}^{2}} \nabla^{x} \nabla^{x^{\top}}\left(G\left(\kappa_{s},|\boldsymbol{x}-\boldsymbol{y}|\right)-G\left(\kappa_{p},|\boldsymbol{x}-\boldsymbol{y}|\right)\right)\right] .
$$

The single- and double-layer potential operators are defined by

$$
\mathcal{S} \boldsymbol{\varphi}=\int_{\Gamma} \Phi(\cdot, \boldsymbol{y}) \boldsymbol{\varphi}(\boldsymbol{y}) d s(\boldsymbol{y}) \text { and } \mathcal{D} \boldsymbol{\psi}=\int_{\Gamma}\left[\boldsymbol{T}_{\boldsymbol{y}} \Phi(\cdot, \boldsymbol{y})\right]^{\top} \boldsymbol{\psi}(\boldsymbol{y}) d s(\boldsymbol{y}),
$$

where $\boldsymbol{T}_{\boldsymbol{y}}=\boldsymbol{T}\left(\boldsymbol{n}(\boldsymbol{y}), \partial_{\boldsymbol{y}}\right)$ and $\boldsymbol{T}_{\boldsymbol{y}} \Phi(\boldsymbol{x}, \boldsymbol{y})$ is the tensor obtained by applying the traction operator $\boldsymbol{T}_{\boldsymbol{y}}$ to each column of $\Phi(\boldsymbol{x}, \boldsymbol{y})$. For a solution $\boldsymbol{u}$ of the Navier equation (1a) in $\Omega^{+}$, that satisfies the Kupradze radiation conditions, one can derive the Somigliana integral representation formula: for $\boldsymbol{x} \in \Omega^{+}$

$$
\boldsymbol{u}(\boldsymbol{x})=\mathcal{D} \boldsymbol{u}_{\mid \Gamma}(\boldsymbol{x})-\mathcal{S} \boldsymbol{t}_{\mid \Gamma}(\boldsymbol{x}) .
$$

The Cauchy data $\left(\boldsymbol{u}_{\mid \Gamma}, \boldsymbol{t}_{\mid \Gamma}\right)$ become the new unknowns of the problem. The displacement field $\boldsymbol{u}$ in $\Omega^{+}$is uniquely determined from the knowledge of these two surface fields.

Given vector densities $\varphi$ and $\psi$, the boundary integral operators $S, D, D^{\prime}$ and $N$ are defined, for $\boldsymbol{x} \in \Gamma$, by

$$
\begin{aligned}
S \boldsymbol{\varphi}(\boldsymbol{x}) & =\int_{\Gamma} \Phi(\boldsymbol{x}, \boldsymbol{y}) \boldsymbol{\varphi}(\boldsymbol{y}) d s(\boldsymbol{y}), \\
D \boldsymbol{\psi}(\boldsymbol{x}) & =\int_{\Gamma}\left[\boldsymbol{T}_{\boldsymbol{y}} \Phi(\boldsymbol{x}, \boldsymbol{y})\right]^{\top} \boldsymbol{\psi}(\boldsymbol{y}) d s(\boldsymbol{y}), \\
D^{\prime} \boldsymbol{\varphi}(\boldsymbol{x}) & =\int_{\Gamma} \boldsymbol{T}_{\boldsymbol{x}}\{\Phi(\boldsymbol{x}, \boldsymbol{y}) \boldsymbol{\varphi}(\boldsymbol{y})\} d s(\boldsymbol{y}), \\
N \boldsymbol{\psi}(\boldsymbol{x}) & =\int_{\Gamma} \boldsymbol{T}_{\boldsymbol{x}}\left\{\left[\boldsymbol{T}_{\boldsymbol{y}} \Phi(\boldsymbol{x}, \boldsymbol{y})\right]^{\top} \boldsymbol{\psi}(\boldsymbol{y})\right\} d s(\boldsymbol{y}) .
\end{aligned}
$$

By applying the exterior Dirichlet and Neumann traces to $\mathcal{S}$ and $\mathcal{D}$ we have [32] 


$$
\begin{aligned}
& (\mathcal{S} \varphi)_{\mid \Gamma}=S \boldsymbol{\varphi}, \quad(\boldsymbol{T S} \boldsymbol{\varphi})_{\mid \Gamma}=\left(-\frac{\mathrm{I}}{2}+D^{\prime}\right) \boldsymbol{\varphi} \\
& (\mathcal{D} \boldsymbol{\psi})_{\mid \Gamma}=\left(\frac{\mathrm{I}}{2}+D\right) \boldsymbol{\psi}, \quad \text { and }(\boldsymbol{T} \mathcal{D} \boldsymbol{\psi})_{\mid \Gamma}=N \boldsymbol{\psi},
\end{aligned}
$$

where I is the identity operator. The operator $S$ is a pseudo-differential operator of order -1 , i.e., it is bounded from $\boldsymbol{H}^{-\frac{1}{2}}(\Gamma)$ to $\boldsymbol{H}^{\frac{1}{2}}(\Gamma)$ and compact from $\boldsymbol{H}^{-\frac{1}{2}}(\Gamma)$ to itself. The operator $D$ and its adjoint $D^{\prime}$ are of order 0 , i.e., they have a strongly singular kernel and are bounded from $\boldsymbol{H}^{\frac{1}{2}}(\Gamma)$ and $\boldsymbol{H}^{-\frac{1}{2}}(\Gamma)$ to themselves, respectively. The operator $N$ is of order 1, i.e., it has a hypersingular kernel and is bounded from $\boldsymbol{H}^{\frac{1}{2}}(\Gamma)$ to $\boldsymbol{H}^{-\frac{1}{2}}(\Gamma)$. The Calderón projectors for the time-harmonic Navier equation are

$$
P^{ \pm}=\left(\begin{array}{cc} 
\pm \frac{\mathrm{I}}{2}+D & -S \\
N & \pm \frac{\mathrm{I}}{2}-D^{\prime}
\end{array}\right) .
$$

We have $P^{+} \circ P^{-}=P^{-} \circ P^{+}=0$ and thus the relations

$$
\begin{gathered}
S D^{\prime}=D S, \quad D^{\prime} N=N D, \\
S N=D^{2}-\frac{\mathrm{I}}{4}, \quad N S=D^{\prime 2}-\frac{\mathrm{I}}{4} .
\end{gathered}
$$

\subsection{Standard boundary integral equations}

There exists various possible boundary integral equations to obtain the Cauchy data $\left(\boldsymbol{u}_{\mid \Gamma}, \boldsymbol{t}_{\mid \Gamma}\right)$. We focus on combined field boundary integral equations because they have the property of unique solvability. The Neumann trace $\boldsymbol{t}_{\mid \Gamma}$ is known through the boundary condition (1b).

We consider a direct method based on the following integral representation formula

$$
\boldsymbol{u}=\mathcal{D}\left(\boldsymbol{u}_{\mid \Gamma}+\boldsymbol{u}_{\mid \Gamma}^{i n c}\right)
$$

Taking the exterior Dirichlet and Neumann traces of the right hand side, we obtain on $\Gamma$

$$
\begin{gathered}
\left(\frac{\mathrm{I}}{2}+D\right)\left(\boldsymbol{u}_{\mid \Gamma}+\boldsymbol{u}_{\mid \Gamma}^{i n c}\right)=\boldsymbol{u}_{\mid \Gamma} \Leftrightarrow\left(\frac{\mathrm{I}}{2}-D\right)\left(\boldsymbol{u}_{\mid \Gamma}+\boldsymbol{u}_{\mid \Gamma}^{i n c}\right)=\boldsymbol{u}_{\mid \Gamma}^{i n c} \quad \text { and } \\
N\left(\boldsymbol{u}_{\mid \Gamma}+\boldsymbol{u}_{\mid \Gamma}^{i n c}\right)=\boldsymbol{t}_{\mid \Gamma}=-\boldsymbol{t}_{\mid \Gamma}^{i n c} .
\end{gathered}
$$

Combining the two previous equations, we construct the standard CFIE: find the physical unknown $\boldsymbol{\psi}=\boldsymbol{u}_{\mid \Gamma}+\boldsymbol{u}_{\mid \Gamma}^{i n c} \in \boldsymbol{H}^{\frac{1}{2}}(\Gamma)$ solution to

$$
\left(\frac{\mathrm{I}}{2}-D-i \eta N\right) \boldsymbol{\psi}=\boldsymbol{u}_{\mid \Gamma}^{i n c}+i \eta \boldsymbol{t}_{\mid \Gamma}^{i n c}, \quad \text { on } \Gamma
$$

with $\eta$ a coupling parameter. The integral Eq. (7) is well-posed for any real positive frequency $\omega$ and any non-zero real parameter $\eta$ [48-50]. However, it involves the boundary integral operator $N$ which is a pseudodifferential operator of order 1 . Thus, this boundary integral equation is of the first-kind and admits a countable set of eigenvalues that tends to infinity. We will see that this standard CFIE is not well-suited for iterative solvers, 
particularly at high frequencies (see Sects. 4 and 5 ). This motivates the research of new and well-conditioned CFIEs.

\section{Analytical preconditioners and regularized CFIE}

\subsection{Principle of analytical preconditioners}

Analytical preconditioning techniques consist in first constructing local approximations of the exact exterior Neumann-to-Dirichlet operator. Then, they are used to get new CFIEs with interesting spectral properties. The exact exterior Neumann-to-Dirichlet operator (called NtD) is defined by

$$
\boldsymbol{V}^{\mathrm{ex}}: \boldsymbol{t}_{\mid \Gamma} \in \boldsymbol{H}^{-\frac{1}{2}}(\Gamma) \mapsto \boldsymbol{V}^{\mathrm{ex}} \boldsymbol{t}_{\mid \Gamma}:=\boldsymbol{u}_{\mid \Gamma} \in \boldsymbol{H}^{\frac{1}{2}}(\Gamma)
$$

and the Somigliana integral representation formula (4) of the scattered field writes

$$
\boldsymbol{u}(\boldsymbol{x})=\mathcal{D} \boldsymbol{u}_{\mid \Gamma}(\boldsymbol{x})-\mathcal{S} \boldsymbol{t}_{\mid \Gamma}(\boldsymbol{x})=\mathcal{D} \boldsymbol{V}^{\mathrm{ex}} \boldsymbol{t}_{\mid \Gamma}(\boldsymbol{x})-\mathcal{S} \boldsymbol{t}_{\mid \Gamma}(\boldsymbol{x}), \boldsymbol{x} \in \Omega^{+} .
$$

Taking the exterior Neumann trace of the representation (9) we obtain

$$
\boldsymbol{t}_{\mid \Gamma}(\boldsymbol{x})=\left(N \boldsymbol{V}^{\mathrm{ex}}+\frac{\mathrm{I}}{2}-D^{\prime}\right) \boldsymbol{t}_{\mid \Gamma}(\boldsymbol{x}), \boldsymbol{x} \in \Gamma,
$$

and hence the exact $\mathrm{NtD}$ operator $\boldsymbol{V}^{\mathrm{ex}}$ satisfies on $\Gamma$

$$
\frac{\mathrm{I}}{2}-D^{\prime}+N V^{\mathrm{ex}}=\mathrm{I} \text {. }
$$

In order to avoid the use of non-physical quantities, we prefer to consider the $L^{2}$-adjoint form of the above-written boundary integral equation

$$
\frac{\mathrm{I}}{2}-D+\boldsymbol{V}^{\mathrm{ex}} N=\mathrm{I},
$$

that is related to the CFIE (7). It then appears that the adjoint NtD $V^{\mathrm{x}^{\prime}}$ is an ideal analytical preconditioner for the CFIE. The use of $\boldsymbol{V}^{\mathrm{e} x^{\prime}}$ (instead of the constant $\eta$ ) to regularize the operator $N$ gives directly the solution to the scattering problem. Furthermore, assume that $\omega$ is not an eigenfrequency of the Navier equation (1a) in $\Omega^{-}$with a Neumann homogeneous boundary condition, we deduce from (10) that the adjoint NtD map is expressed in terms of elementary boundary integral operators on $\Gamma$ by

$$
\boldsymbol{V}^{\mathrm{ex}^{\prime}}=\left(\frac{\mathrm{I}}{2}+D\right) N^{-1}
$$

The Calderón formula $S N=D^{2}-\frac{\mathrm{I}}{4}$ (see (6)) leads to another integral representation

$$
\boldsymbol{V}^{\mathrm{ex}^{\prime}}=-\left(\frac{\mathrm{I}}{2}-D\right)^{-1} S
$$

However, it is numerically too expensive to apply one of these representations of the operator $\boldsymbol{V}^{\mathrm{ex}^{\prime}}$ as a preconditioner for the CFIE. Instead, an approximation $\boldsymbol{V}^{\prime}$ of $\boldsymbol{V}^{\mathrm{ex}^{\prime}}$ is introduced to construct a preconditioned CFIE : Find the total field $\boldsymbol{\psi}=\boldsymbol{u}_{\mid \Gamma}+\boldsymbol{u}_{\mid \Gamma}^{\text {inc }} \in \boldsymbol{H}^{\frac{1}{2}}(\Gamma)$ solution to 


$$
\left(\frac{\mathrm{I}}{2}-D+\boldsymbol{V}^{\prime} N\right) \boldsymbol{\psi}=\boldsymbol{u}_{\mid \Gamma}^{i n c}-\boldsymbol{V}^{\prime} \boldsymbol{t}_{\mid \Gamma}^{i n c}, \quad \text { on } \Gamma .
$$

The spectral properties of (13) depend on the choice of the approximate adjoint NtD map $\boldsymbol{V}^{\prime}$. The idea is to consider only the principal part of the exact adjoint operator $\boldsymbol{V}^{\text {ex }}$. It reads

$$
\boldsymbol{V}^{\prime}=-\left(\frac{\mathrm{I}}{2}-P(D)\right)^{-1} P(S)
$$

where the operators $P(S)$ and $P(D)$ are respectively the principal parts of the boundary integral operators $S$ and $D$.

\subsection{Three-dimensional case}

In this subsection, we give the expressions of different approximations of the adjoint NtD map and their corresponding preconditioned CFIEs. First, we need to introduce the expressions of the principal parts $P(S)$ and $P(D)$ of the operators $S$ and $D$ using tools proposed in [32] (see Lemmas 3.2 and 3.3 respectively and their proofs in [32]). The operator $P(S)$ is decomposed into two terms: $P(S)=S_{1}+S_{2}$ with

$$
\begin{aligned}
& S_{1}=\frac{i}{2 \rho \omega^{2}}\left(\boldsymbol{n}\left(\Delta_{\Gamma}+\kappa_{p}^{2} \mathrm{I}\right)^{\frac{1}{2}} \boldsymbol{n} \cdot \mathrm{I}_{\boldsymbol{n}}+\left(\boldsymbol{\Delta}_{\Gamma}+\kappa_{s}^{2} \mathrm{I}\right)^{-\frac{1}{2}}\left(\kappa_{s}^{2} \mathrm{I}_{\boldsymbol{t}}+\nabla_{\Gamma} \operatorname{div}_{\Gamma} \mathrm{I}_{\tau}\right)\right) \\
& S_{2}=-\frac{i}{2 \rho \omega^{2}}\left(\boldsymbol{n} \Delta_{\Gamma}\left(\Delta_{\Gamma}+\kappa_{s}^{2} \mathrm{I}\right)^{-\frac{1}{2}} \boldsymbol{n} \cdot \mathrm{I}_{\boldsymbol{n}}+\nabla_{\Gamma}\left(\Delta_{\Gamma}+\kappa_{p}^{2} \mathrm{I}\right)^{-\frac{1}{2}} \operatorname{div}_{\Gamma} \mathrm{I}_{\tau}\right)
\end{aligned}
$$

where $\mathrm{I}_{\boldsymbol{n}}=\boldsymbol{n} \otimes \boldsymbol{n}$ and $\mathrm{I}_{\boldsymbol{\tau}}=\mathrm{I}-\mathrm{I}_{\boldsymbol{n}}$. We refer to [54, pages 68-75] for the definition of the surface differential operators: the tangential gradient $\nabla_{\Gamma}$, the surface divergence $\operatorname{div}_{\Gamma}$ and the scalar Laplace-Beltrami operator $\Delta_{\Gamma}$. The square-root $z^{1 / 2}$ of a complex number $z$ stands for the classical complex square-root with branch-cut along the negative real axis. The operator $P(D)$ is also decomposed into two terms: $P(D)=D_{1}+D_{2}$ with

$$
\begin{aligned}
D_{1}= & \frac{i}{2}\left(\boldsymbol{n}\left(\Delta_{\Gamma}+\kappa_{p}^{2} \mathrm{I}\right)^{-\frac{1}{2}} \operatorname{div}_{\Gamma} \mathrm{I}_{\tau}-\nabla_{\Gamma}\left(\Delta_{\Gamma}+\kappa_{s}^{2} \mathrm{I}\right)^{-\frac{1}{2}} \boldsymbol{n} \cdot \mathrm{I}_{\boldsymbol{n}}\right) \\
D_{2}= & \frac{i \mu}{\rho \omega^{2}}\left(-\boldsymbol{n}\left(\Delta_{\Gamma}+\kappa_{s}^{2} \mathrm{I}\right)^{\frac{1}{2}} \operatorname{div}_{\Gamma} \mathrm{I}_{\tau}+\boldsymbol{n} \Delta_{\Gamma}\left(\Delta_{\Gamma}+\kappa_{p}^{2} \mathrm{I}\right)^{-\frac{1}{2}} \operatorname{div}_{\Gamma} \mathrm{I}_{\tau}\right. \\
& \left.+\nabla_{\Gamma}\left(\Delta_{\Gamma}+\kappa_{p}^{2} \mathrm{I}\right)^{\frac{1}{2}}\left(\boldsymbol{n} \cdot \mathrm{I}_{\boldsymbol{n}}\right)-\nabla_{\Gamma}\left(\Delta_{\Gamma}+\kappa_{s}^{2} \mathrm{I}\right)^{-\frac{1}{2}} \Delta_{\Gamma}\left(\boldsymbol{n} \cdot \mathrm{I}_{\boldsymbol{n}}\right)\right) .
\end{aligned}
$$

From (12)-(15), (16), we derive several approximations of the adjoint NtD map.

Low-order approximation. The low-order expression of the adjoint NtD map $\boldsymbol{V}^{\mathrm{ex}^{\prime}}$ comes from major approximations. First, we keep in (14) only the contribution of $P(S)$ (i.e., $V^{\prime} \approx-2 P(S)$ ). Then, we replace in (15) the surface differential operators by their first eigenvalues, equal to zero. Using the relations between the wavenumbers and the Lamé parameters (see Sect. 2.1), we obtain the following adjoint NtD approximation

$$
\boldsymbol{V}^{\prime}:=\boldsymbol{V}_{\mathrm{LO}}^{\prime}=-i\left(\frac{1}{(\lambda+2 \mu) \kappa_{p}} \mathrm{I}_{\boldsymbol{n}}+\frac{1}{\mu \kappa_{s}} \mathrm{I}_{t}\right) .
$$

Importantly, it results from these simplifications that the operator (17) is only coming from $S_{1}$ (15). This low-order approximation is the equivalent in elasticity of the zeroth-order 
approximation $1 /(i \kappa)$ of the acoustic $\mathrm{NtD}$ map (where $\kappa$ is the wavenumber). The associated preconditioned integral equation is given by

$$
\left(\frac{\mathrm{I}}{2}-D+\boldsymbol{V}_{\mathrm{LO}}^{\prime} N\right) \boldsymbol{\psi}=\boldsymbol{u}_{\mid \Gamma}^{i n c}-\boldsymbol{V}_{\mathrm{LO}}^{\prime} \boldsymbol{t}_{\mid \Gamma}^{i n c}, \quad \text { on } \Gamma
$$

and is called LO-preconditioned CFIE (LO P-CFIE) in the remaining of this paper. This new boundary integral equation is the counterpart in elasticity of the usual CFIE with the optimal coupling parameter of Kress [46] in acoustics. The main advantage of this preconditioner is to be very easy to implement. By construction, the approximation (17) provides a good clustering of the first eigenvalues (associated with propagating modes) only. We will show that we need high-order approximations to regularize the operator $N$ of order 1 and then to cluster eigenvalues associated to evanescent modes (see Sects. 4 and 5).

High-order approximation. Similarly to previous works [22, 32] for the Dirichlet boundary conditions, we propose to consider the following two high-order approximations of the adjoint $\mathrm{NtD}$ map

$$
\begin{gathered}
\boldsymbol{V}_{\mathrm{HO}(1)}^{\prime}=-2 P(S) \\
\boldsymbol{V}_{\mathrm{HO}(2)}^{\prime}=-\left(\frac{\mathrm{I}}{2}-P(D)\right)^{-1} P(S) .
\end{gathered}
$$

Due to numerical considerations, these approximations are preferred to (11) that involves the principal part of the operator $N^{-1}$. In the approximation (19), the contribution of the double-layer boundary integral operator $D$ is not taking into account and thus avoids an operator inversion. It corresponds to the NtD choice usually done in acoustics [4-6] and electromagnetics $[30,33]$. However, the operator $D$ is not compact in elasticity, even for smooth surfaces. Thus, we also consider an alternative that takes into account the contribution of its principal part in (20). We will observe in the sequel how it impacts the spectral behavior of the preconditioned CFIE (13). We get the corresponding two P-CFIEs:

- the High-Order preconditioned CFIE with one term (HO(1) P-CFIE): integral Eq. (13) and the analytical preconditioner $V^{\prime}:=V_{\mathrm{HO}(1)}^{\prime}(19)$ without the contribution $P(D)$ associated with the double-layer boundary integral operator.

- the High-Order preconditioned CFIE with two terms (HO(2) P-CFIE): integral Eq. (13) and the analytical preconditioner $\boldsymbol{V}^{\prime}:=\boldsymbol{V}_{\mathrm{HO}(2)}^{\prime}(20)$ with the principal part $P(D)$.

The preconditioner $\boldsymbol{V}^{\prime}$ contains square-root operators of the form $\left(\Delta_{\Gamma}+\kappa_{\gamma}^{2} \mathrm{I}\right)^{1 / 2}$ with $\gamma=$ $s, p$ and their inverses. An artificial singularity of the square-root operator appears in the transition zone from the propagating modes to the evanescent ones. The presence of this singularity yields a wrong representation of the grazing modes. To model the behavior in the transition zone, we use a regularization [6] by adding a small local damping parameter $\varepsilon_{\gamma}>0$ to the wavenumber $\kappa_{\gamma}$. We set $\kappa_{\gamma, \varepsilon}:=\kappa_{\gamma}+i \varepsilon_{\gamma}$ and we consider square-root operators $\left(\Delta_{\Gamma}+\kappa_{\gamma, \varepsilon}^{2} \mathrm{I}\right)^{1 / 2}$ and their inverses in the preconditioner $\boldsymbol{V}^{\prime}$. We denote by $P_{\varepsilon}(S)$ and $P_{\varepsilon}(D)$ the corresponding principal parts. The addition of a local damping is important to obtain the well-posed character of the corresponding preconditioned CFIEs.

The main subject of this work concerns the implementation and study of three possible approximations of $\boldsymbol{V}^{\prime}$. They are discussed in the next sections. For existence and uniqueness results, we refer to [32] for details but the main result is given in the following proposition. 
Proposition 1 Consider $\Gamma$ a smooth surface, the P-CFIEs (19) and (20) are uniquely solvable in $H^{1 / 2}(\Gamma)$ for any positive real wavenumber and any damping parameter $\varepsilon \neq 0$.

The proof relies on two simple ingredients [32]: the symbolic calculus and the Fredholm alternative. Indeed, the operator $\boldsymbol{V}^{\prime}$ has the required regularizing effect on the operator $N$ and the operators in the P-CFIEs (19) and (20) are Fredholm operators of index zero. The associated interior Robin problem is the same as for the Dirichlet problem. Then injectivity follows from the positive imaginary part of $\left(\boldsymbol{V}^{\prime}\right)^{-1}$ (see [32, proof of theorem 3.8]). We conclude with Riesz theory. The case of Lipschitz boundaries would require more investigation which is out of the scope of this work.

\subsection{Two-dimensional case}

The main difference between 2D and 3D for the proposed preconditioned CFIEs lies in the expressions of the principal parts $P(S)$ and $P(D)$. We denote by $s$ the anticlockwise directed curvilinear abscissa along $\Gamma$. We introduce the curvilinear derivative $\partial_{s}$. The LaplaceBeltrami operator over $\Gamma$ is defined by $\Delta_{\Gamma}:=\partial_{s}^{2}$. Furthermore, we use the relations $\nabla_{\Gamma} u=$ $\tau \partial_{s} u$ and $\operatorname{div}_{\Gamma} \boldsymbol{u}=\partial_{s}(\tau \cdot \boldsymbol{u})$. We deduce that in $2 \mathrm{D}$ we have $P(S)=S_{1}+S_{2}$ with

$$
\begin{aligned}
& S_{1}=\frac{i}{2 \rho \omega^{2}}\left(\boldsymbol{n}\left(\Delta_{\Gamma}+\kappa_{p, \varepsilon}^{2} \mathrm{I}\right)^{\frac{1}{2}} \boldsymbol{n} \cdot \mathrm{I}_{\boldsymbol{n}}+\boldsymbol{\tau}\left(\Delta_{\Gamma}+\kappa_{s, \varepsilon}^{2} \mathrm{I}\right)^{\frac{1}{2}} \boldsymbol{\tau} \cdot \mathrm{I}_{\tau}\right) \\
& S_{2}=-\frac{i}{2 \rho \omega^{2}}\left(\boldsymbol{n}\left(\Delta_{\Gamma}+\kappa_{s, \varepsilon}^{2} \mathrm{I}\right)^{-\frac{1}{2}} \Delta_{\Gamma}\left(\boldsymbol{n} \cdot \mathrm{I}_{\boldsymbol{n}}\right)+\boldsymbol{\tau}\left(\Delta_{\Gamma}+\kappa_{p, \varepsilon}^{2} \mathrm{I}\right)^{-\frac{1}{2}} \Delta_{\Gamma}\left(\boldsymbol{\tau} \cdot \mathrm{I}_{\tau}\right)\right)
\end{aligned}
$$

and $P(D)=D_{1}+D_{2}$ with

$$
\begin{aligned}
D_{1}= & \frac{i}{2}\left(\boldsymbol{n} \partial_{s}\left(\Delta_{\Gamma}+\kappa_{p, \varepsilon}^{2} \mathrm{I}\right)^{-\frac{1}{2}} \tau \cdot \mathrm{I}_{\tau}-\tau \partial_{s}\left(\Delta_{\Gamma}+\kappa_{s, \varepsilon}^{2} \mathrm{I}\right)^{-\frac{1}{2}} \boldsymbol{n} \cdot \mathrm{I}_{\boldsymbol{n}}\right) \\
D_{2}= & \frac{i \mu}{\rho \omega^{2}}\left(-\boldsymbol{n} \partial_{s}\left(\Delta_{\Gamma}+\kappa_{s, \varepsilon}^{2} \mathrm{I}\right)^{\frac{1}{2}} \tau \cdot \mathrm{I}_{\tau}+\boldsymbol{n} \partial_{s}\left(\Delta_{\Gamma}+\kappa_{p, \varepsilon}^{2} \mathrm{I}\right)^{-\frac{1}{2}} \Delta_{\Gamma}\left(\tau \cdot \mathrm{I}_{\tau}\right)\right. \\
& \left.+\tau \partial_{s}\left(\Delta_{\Gamma}+\kappa_{p, \varepsilon}^{2} \mathrm{I}\right)^{\frac{1}{2}}\left(\boldsymbol{n} \cdot \mathrm{I}_{\boldsymbol{n}}\right)-\tau \partial_{s}\left(\Delta_{\Gamma}+\kappa_{s, \varepsilon}^{2} \mathrm{I}\right)^{-\frac{1}{2}} \Delta_{\Gamma}\left(\boldsymbol{n} \cdot \mathrm{I}_{\boldsymbol{n}}\right)\right) .
\end{aligned}
$$

\section{Spectral study for the spherical case}

Once the three possible preconditioned CFIEs presented, we perform an analytical investigation of the eigenvalue clustering of these CFIE operators for the 3D spherical case. We also compare the stability of their condition numbers with respect to the mesh refinement and frequency increase. We use some results obtained in [32, Section 4 and Appendix B] for the three-dimensional Dirichlet boundary condition case.

\subsection{Asymptotic analysis}

Let $\mathbf{S}^{2}$ be the unit sphere in $\mathbb{R}^{3}$ with outward unit normal vector denoted by $\hat{\boldsymbol{x}}$. We introduce the scalar spherical harmonics $Y_{\ell j}$ of order $\ell \in \mathbb{N}$ and degree $j=-\ell, \ldots, \ell$. The concatenation of the tangential vector spherical harmonics defined for $\ell \in \mathbb{N}^{*}$ by 


$$
\mathcal{Y}_{\ell j}^{(1)}=(\ell(\ell+1))^{-\frac{1}{2}} \nabla_{\mathbf{S}^{2}} Y_{\ell j}, \quad \mathcal{Y}_{\ell j}^{(2)}=(\ell(\ell+1))^{-\frac{1}{2}} \nabla_{\mathbf{S}^{2}} Y_{\ell j} \times \hat{\boldsymbol{x}}
$$

with the radial spherical harmonics defined for $\ell \in \mathbb{N}$ by $\mathcal{Y}_{\ell j}^{(3)}=\hat{\boldsymbol{x}} Y_{\ell j}$, forms an orthonormal basis function of $\boldsymbol{H}^{\frac{1}{2}}\left(\mathbf{S}^{2}\right)$. In the case of an elastic sphere of radius $R$, the boundary integral operators $D$ and $N$ can be expanded in this basis function both such that $C \mathcal{Y}_{0,0}^{(3)}=C_{0}^{(3,3)} \mathcal{Y}_{0,0}^{(3)}$ and for $\ell \geq 1$

$$
C\left(\begin{array}{c}
\mathcal{Y}_{\ell, j}^{(1)} \\
\mathcal{Y}_{\ell, j}^{(2)} \\
\mathcal{Y}_{\ell, j}^{(3)}
\end{array}\right)=\left(\begin{array}{ccc}
C_{\ell}^{(1,1)} & 0 & C_{\ell}^{(1,3)} \\
0 & C_{\ell}^{(2,2)} & 0 \\
C_{\ell}^{(3,1)} & 0 & C_{\ell}^{(3,3)}
\end{array}\right)\left(\begin{array}{c}
\mathcal{Y}_{\ell, j}^{(1)} \\
\mathcal{Y}_{\ell, j}^{(2)} \\
\mathcal{Y}_{\ell, j}^{(3)}
\end{array}\right)
$$

where $C=D$ or $N$. In the high frequency-regime, we distinguish three zones of modes: the hyperbolic zone for $\ell \ll \kappa_{p} R$ (propagating modes), the elliptic zone when $\ell \gg \kappa_{s} R$ (evanescent modes), and the transition zone of physical surface modes between $\ell \approx \kappa_{p} R$ and $\ell \approx \kappa_{s} R$. First, let us consider the hyperbolic zone. We obtain the following asymptotic behavior.

Proposition 2 When $\kappa_{p} R \rightarrow \infty$ and $\kappa_{s} R \rightarrow \infty$ we have

- for the operator D:

$$
\begin{array}{ll}
D_{\ell}^{(1,1)}=\frac{1}{2}-\sin ^{2}\left(\kappa_{s} R-(\ell+1) \frac{\pi}{2}\right)+\frac{i}{2} \sin \left(2\left(\kappa_{s} R-(\ell+1) \frac{\pi}{2}\right)\right)+\mathcal{O}\left(\frac{1}{\kappa_{s} R}\right), \quad \ell \geq 1, \\
D_{\ell}^{(2,2)}=\frac{1}{2}-\cos ^{2}\left(\kappa_{s} R-(\ell+1) \frac{\pi}{2}\right)-\frac{i}{2} \sin \left(2\left(\kappa_{s} R-(\ell+1) \frac{\pi}{2}\right)\right)+\mathcal{O}\left(\frac{1}{\kappa_{s} R}\right), \quad \ell \geq 1, \\
D_{\ell}^{(3,3)}=\frac{1}{2}-\sin ^{2}\left(\kappa_{p} R-(\ell+1) \frac{\pi}{2}\right)+\frac{i}{2} \sin \left(2\left(\kappa_{p} R-(\ell+1) \frac{\pi}{2}\right)\right)+\mathcal{O}\left(\frac{1}{\kappa_{p} R}\right), \quad \ell \geq 0, \\
D_{\ell}^{(1,3)}=\mathcal{O}\left(\frac{1}{\kappa_{s} R}\right), \quad D_{\ell}^{(3,1)}=\mathcal{O}\left(\frac{1}{\kappa_{s} R}\right), \quad \ell \geq 1,
\end{array}
$$

- and for the operator $N$ :

$$
\begin{aligned}
N_{\ell}^{(1,1)}= & \frac{\mu \kappa_{s}}{i}\left(-\cos ^{2}\left(\kappa_{s} R-(\ell+1) \frac{\pi}{2}\right)-\frac{i}{2} \sin \left(2\left(\kappa_{s} R-(\ell+1) \frac{\pi}{2}\right)\right)\right) \\
& +\mathcal{O}\left(\frac{1}{\kappa_{s} R}\right), \quad \ell \geq 1, \\
N_{\ell}^{(2,2)}= & \frac{\mu \kappa_{s}}{i}\left(-\sin ^{2}\left(\kappa_{s} R-(\ell+1) \frac{\pi}{2}\right)+\frac{i}{2} \sin \left(2\left(\kappa_{s} R-(\ell+1) \frac{\pi}{2}\right)\right)\right) \\
& +\mathcal{O}\left(\frac{1}{\kappa_{s} R}\right), \quad \ell \geq 1, \\
N_{\ell}^{(3,3)}= & i(\lambda+2 \mu) \kappa_{p}\left(\cos ^{2}\left(\kappa_{p} R-(\ell+1) \frac{\pi}{2}\right)+\frac{i}{2} \sin \left(2\left(\kappa_{p} R-(\ell+1) \frac{\pi}{2}\right)\right)\right) \\
& +\mathcal{O}\left(\frac{1}{\kappa_{p} R}\right), \quad \ell \geq 0, \\
N_{\ell}^{(1,3)}= & \mathcal{O}\left(\frac{1}{\kappa_{s} R}\right), \quad N_{\ell}^{(3,1)}=\mathcal{O}\left(\frac{1}{\kappa_{s} R}\right), \quad \ell \geq 1 .
\end{aligned}
$$


From these results and the integral representation(11), we retrieve the low-order approximation (17) of the $\mathrm{NtD}$ operator

$$
V_{\mathrm{LO}}^{\prime}=\left(\begin{array}{ccc}
\frac{1}{i \mu \kappa_{s}} & 0 & 0 \\
0 & \frac{1}{i \mu \kappa_{s}} & 0 \\
0 & 0 & \frac{1}{i(\lambda+2 \mu) \kappa_{p}}
\end{array}\right)
$$

so that in the hyperbolic zone (when $\kappa_{s} R \rightarrow \infty$ ) we get

$$
\frac{\mathrm{I}}{2}-D+V_{\mathrm{LO}}^{\prime} N=\mathrm{I}+\mathcal{O}\left(\frac{1}{\kappa_{S} R}\right) .
$$

The high-order P-CFIE operators share also this good property. Let us observe now the behaviour of the different operators in the elliptic zone. The following asymptotic results hold.

Proposition 3 When $\ell \rightarrow \infty$, we have

- for the operator D:

$$
\begin{aligned}
& D_{\ell}^{(1,1)}=-\frac{\mu}{\lambda+2 \mu} \frac{1}{2(2 \ell+1)}+\mathcal{O}\left(\frac{1}{\ell^{3}}\right), \\
& D_{\ell}^{(2,2)}=-\frac{3}{2(2 \ell+1)}+O\left(\frac{1}{\ell^{3}}\right), \\
& D_{\ell}^{(3,3)}=-\frac{3 \mu}{\lambda+2 \mu} \frac{1}{2(2 \ell+1)}+\mathcal{O}\left(\frac{1}{\ell^{3}}\right), \\
& D_{\ell}^{(1,3)}=D_{\ell}^{(3,1)}=\frac{\mu}{2(\lambda+2 \mu)}+\mathcal{O}\left(\frac{1}{\ell^{2}}\right),
\end{aligned}
$$

- and for the operator $N$ :

$$
\begin{aligned}
& N_{\ell}^{(1,1)}=\frac{-2 \mu(\lambda+\mu)}{\lambda+2 \mu} \frac{\ell+\frac{1}{2}}{2 R}+\mathcal{O}\left(\frac{1}{\ell}\right), \\
& N_{\ell}^{(2,2)}=-\mu \frac{\ell+\frac{1}{2}}{2 R}+\mathcal{O}\left(\frac{1}{\ell}\right), \\
& N_{\ell}^{(3,3)}=\frac{-2 \mu(\lambda+\mu)}{\lambda+2 \mu} \frac{\ell+\frac{1}{2}}{2 R}+\mathcal{O}\left(\frac{1}{\ell}\right), \\
& N_{\ell}^{(1,3)}=N_{\ell}^{(3,1)}=\frac{\mu(3 \lambda+\mu)}{2 R(\lambda+2 \mu)}+\mathcal{O}\left(\frac{1}{\ell^{2}}\right) .
\end{aligned}
$$

The asymptotic behavior for large modes of the boundary integral operator $N$ are in accordance with the fact that the standard CFIE operator is of the first kind. The application of the low-order approximation $\boldsymbol{V}_{\mathrm{LO}}^{\prime}(23)$ is not sufficient to regularize the operator $N$ in the elliptic part. It does not provide an eigenvalue clustering in this zone (see Fig. 1). Highorder approximations are needed to efficiently regularize the operator $N$. We have 


$$
\boldsymbol{V}_{\mathrm{HO}(2)}^{\prime}=-\left(\frac{\mathrm{I}}{2}-P_{\varepsilon}(D)\right)^{-1} P_{\varepsilon}(S)
$$

where

$$
P_{\varepsilon}(S)\left(\begin{array}{c}
\mathcal{Y}_{\ell, j}^{(1)} \\
\mathcal{Y}_{\ell, j}^{(2)} \\
\mathcal{Y}_{\ell, j}^{(3)}
\end{array}\right)=\left(\begin{array}{ccc}
s_{\ell, \varepsilon}^{(1,1)} & 0 & 0 \\
0 & s_{\ell, \varepsilon}^{(2,2)} & 0 \\
0 & 0 & s_{\ell, \varepsilon}^{(3,3)}
\end{array}\right)\left(\begin{array}{c}
\mathcal{Y}_{\ell, j}^{(1)} \\
\mathcal{Y}_{\ell, j}^{(2)} \\
\mathcal{Y}_{\ell, j}^{(3)}
\end{array}\right)
$$

with asymptotics when $\ell \rightarrow \infty$

$$
\begin{aligned}
& s_{\ell, \varepsilon}^{(1,1)}=\frac{i}{2 \rho \omega^{2}}\left[\left(\kappa_{s, \varepsilon}^{2}-\frac{\ell(\ell+1)}{R^{2}}\right)^{\frac{1}{2}}+\frac{\ell(\ell+1)}{R^{2}}\left(\kappa_{p, \varepsilon}^{2}-\frac{\ell(\ell+1)}{R^{2}}\right)^{-\frac{1}{2}}\right]=\frac{\left(\kappa_{s, \varepsilon}^{2}+\kappa_{p, \varepsilon}^{2}\right) R}{4 \rho \omega^{2} \sqrt{\ell(\ell+1)}}+o\left(\ell^{-1}\right), \\
& s_{\ell, \varepsilon}^{(2,2)}=\frac{i}{2 \rho \omega^{2}} \kappa_{s, \varepsilon}^{2}\left(\kappa_{s, \varepsilon}^{2}-\frac{\ell(\ell+1)}{R^{2}}\right)^{-\frac{1}{2}}=\frac{\kappa_{s, \varepsilon}^{2} R}{2 \rho \omega^{2} \sqrt{\ell(\ell+1)}}+o\left(\ell^{-1}\right), \\
& s_{\ell, \varepsilon}^{(3,3)}=\frac{i}{2 \rho \omega^{2}}\left[\left(\kappa_{p}^{2}-\frac{\ell(\ell+1)}{R^{2}}\right)^{\frac{1}{2}}+\frac{\ell(\ell+1)}{R^{2}}\left(\kappa_{s, \varepsilon}^{2}-\frac{\ell(\ell+1)}{R^{2}}\right)^{-\frac{1}{2}}\right]=\frac{\left(\kappa_{s, \varepsilon}^{2}+\kappa_{p, \varepsilon}^{2}\right) R}{4 \rho \omega^{2} \sqrt{\ell(\ell+1)}}+o\left(\ell^{-1}\right),
\end{aligned}
$$

and

$$
P_{\varepsilon}(D)\left(\begin{array}{c}
\mathcal{Y}_{\ell, j}^{(1)} \\
\mathcal{Y}_{\ell, j}^{(2)} \\
\mathcal{Y}_{\ell, j}^{(3)}
\end{array}\right)=\left(\begin{array}{ccc}
0 & 0 & d_{\ell, \varepsilon}^{(1,3)} \\
0 & 0 & 0 \\
d_{\ell, \varepsilon}^{(3,1)} & 0 & 0
\end{array}\right)\left(\begin{array}{c}
\mathcal{Y}_{\ell, j}^{(1)} \\
\mathcal{Y}_{\ell, j}^{(2)} \\
\mathcal{Y}_{\ell, j}^{(3)}
\end{array}\right)
$$

with asymptotics when $\ell \rightarrow \infty$

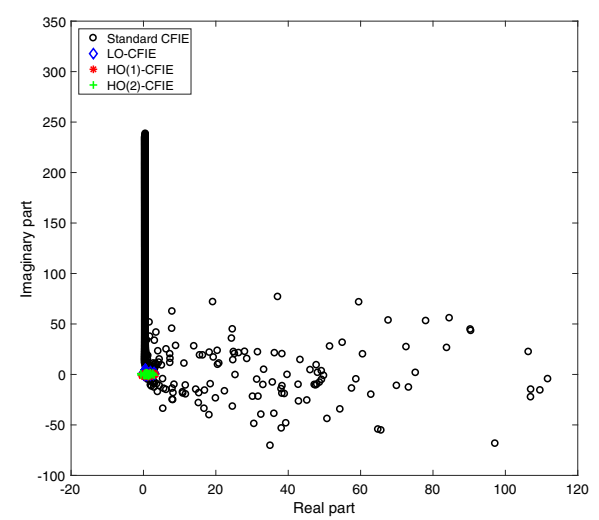

(a)

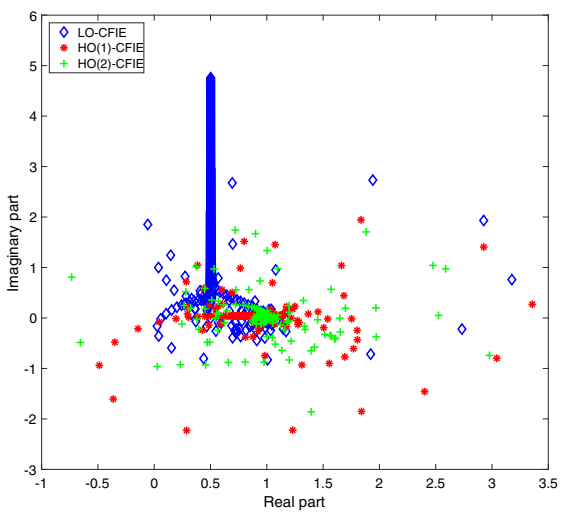

(b)

Fig. 1 Unit sphere. Distribution of the eigenvalues of the standard and three P-CFIEs $\left(\eta=1, \kappa_{s}=\omega=50\right.$ and $n_{\lambda_{s}}=12$ ). a Complete distribution and $\mathbf{b}$ Closer view on the clustering 


$$
\begin{aligned}
d_{\ell, \varepsilon}^{(1,3)}= & \frac{i}{\kappa_{s, \varepsilon}^{2}}\left(\frac{\ell(\ell+1)}{R^{2}}\right)^{1 / 2}\left[\left(\kappa_{s, \varepsilon}^{2}-\frac{\ell(\ell+1)}{R^{2}}\right)^{\frac{1}{2}}+\frac{\ell(\ell+1)}{R^{2}}\left(\kappa_{p, \varepsilon}^{2}-\frac{\ell(\ell+1)}{R^{2}}\right)^{-\frac{1}{2}}\right] \\
& -\frac{i}{2}\left(\frac{\ell(\ell+1)}{R^{2}}\right)^{1 / 2}\left(\kappa_{p, \varepsilon}^{2}-\frac{\ell(\ell+1)}{R^{2}}\right)^{-\frac{1}{2}}=\frac{\kappa_{p, \varepsilon}^{2}}{2 \kappa_{s, \varepsilon}^{2}}+o(1), \\
d_{\ell, \varepsilon}^{(3,1)}= & \frac{i}{\kappa_{s, \varepsilon}^{2}}\left(\frac{\ell(\ell+1)}{R^{2}}\right)^{1 / 2}\left[\left(\kappa_{p, \varepsilon}^{2}-\frac{\ell(\ell+1)}{R^{2}}\right)^{\frac{1}{2}}+\frac{\ell(\ell+1)}{R^{2}}\left(\kappa_{s, \varepsilon}^{2}-\frac{\ell(\ell+1)}{R^{2}}\right)^{-\frac{1}{2}}\right] \\
& -\frac{i}{2}\left(\frac{\ell(\ell+1)}{R^{2}}\right)^{1 / 2}\left(\kappa_{s, \varepsilon}^{2}-\frac{\ell(\ell+1)}{R^{2}}\right)^{-\frac{1}{2}}=\frac{\kappa_{p, \varepsilon}^{2}}{2 \kappa_{s, \varepsilon}^{2}}+o(1) .
\end{aligned}
$$

Thus, we obtain the following behaviour of the high-order approximate NtD map when $\ell \rightarrow \infty$

$$
\boldsymbol{V}_{\mathrm{HO}(2)}^{\prime}\left(\begin{array}{l}
\mathcal{Y}_{\ell, j}^{(1)} \\
\boldsymbol{\mathcal { Y }}_{\ell, j}^{(2)} \\
\boldsymbol{\mathcal { Y }}_{\ell, j}^{(3)}
\end{array}\right)=-\frac{R}{\sqrt{\ell(\ell+1)}}\left(\begin{array}{cccc}
\frac{1}{2 \rho \omega^{2}} \frac{\kappa_{s, \varepsilon}^{4}}{\kappa_{s, \varepsilon}^{2}-\kappa_{p, \varepsilon}^{2}} & 0 & \frac{1}{2 \rho \omega^{2}} \frac{\kappa_{s, \varepsilon}^{2} \kappa_{p, \varepsilon}^{2}}{\kappa_{s, \varepsilon}^{2}-\kappa_{p, \varepsilon}^{2}} \\
0 & \frac{\kappa_{s, \varepsilon}^{2}}{\rho \omega^{2}} & 0 \\
\frac{1}{2 \rho \omega^{2}} \frac{\kappa_{s, \varepsilon}^{2} \kappa_{p, \varepsilon}^{2}}{\kappa_{s, \varepsilon}^{2}-\kappa_{p, \varepsilon}^{2}} & 0 & \frac{1}{2 \rho \omega^{2}} \frac{\kappa_{s, \varepsilon}^{4}}{\kappa_{s, \varepsilon}^{2}-\kappa_{p, \varepsilon}^{2}}
\end{array}\right)\left(\begin{array}{cc}
\mathcal{Y}_{\ell, j}^{(1)} & \\
& \mathcal{Y}_{\ell, j}^{(2)} \\
\mathcal{Y}_{\ell, j}^{(3)} &
\end{array}\right)+o\left(\ell^{-1}\right)
$$

and of the corresponding $\mathrm{HO}(2) \mathrm{P}-\mathrm{CFIE}$ operator

$$
\left.\begin{array}{l}
\left(\frac{\mathrm{I}}{2}-D+\boldsymbol{V}_{\mathrm{HO}(2)}^{\prime} N\right)\left(\begin{array}{c}
\boldsymbol{Y}_{\ell, j}^{(1)} \\
\boldsymbol{Y}_{\ell, j}^{(2)} \\
\boldsymbol{Y}_{\ell, j}^{(3)}
\end{array}\right) \\
=\left(\begin{array}{ccc}
\frac{1}{2}+\frac{\kappa_{s, \varepsilon}^{4}\left(\kappa_{s}^{2}-\kappa_{p}^{2}\right)}{2 \kappa_{s}^{4}\left(\kappa_{s, \varepsilon}^{2}-\kappa_{p, \varepsilon}^{2}\right)} & \frac{\mu}{2(\lambda+2, \mu)}\left(\frac{\kappa_{s, \varepsilon}^{2} \kappa_{p, \varepsilon}^{2}\left(\kappa_{s}^{2}-\kappa_{p}^{2}\right)}{\kappa_{s}^{2} \kappa_{p}^{2}\left(\kappa_{s, \varepsilon}^{2}-\kappa_{p, \varepsilon}^{2}\right)}-1\right) \\
0 & \frac{1}{2}+\frac{\kappa_{s, \varepsilon}^{2}}{2 \kappa_{s}^{2}} & 0 \\
\frac{\mu}{2(\lambda+2, \mu)}\left(\frac{\kappa_{s, 2}^{2} \kappa_{p, \varepsilon}^{2}\left(\kappa_{s}^{2}-\kappa_{p}^{2}\right)}{\kappa_{s}^{2} \kappa_{p}^{2}\left(\kappa_{s, \varepsilon}^{2}-\kappa_{p, \varepsilon}^{2}\right)}-1\right) & 0 & \frac{1}{2}+\frac{\kappa_{s, \varepsilon}^{4}\left(\kappa_{s}^{2}-\kappa_{p}^{2}\right)}{2 \kappa_{s}^{4}\left(\kappa_{s, \varepsilon}^{2}-\kappa_{p, \varepsilon}^{2}\right)}
\end{array}\right)\left(\begin{array}{ll}
\mathcal{Y}_{\ell, j}^{(1)} & \\
\mathcal{Y}_{\ell, j}^{(3)} &
\end{array}\right)+o\left(\ell^{-1}\right) . \\
\mathcal{Y}_{\ell, j}^{(2)}
\end{array}\right) .
$$

This result shows that the eigenvalues of the $\mathrm{HO}(2)$-CFIE operator are well-clustered around the unit in the elliptic zone. This is also the case for the $\mathrm{HO}(1)$-CFIE operator.

\subsection{Illustration of the spectral properties for the unit sphere}

We now consider a finite dimensional approximation. We keep only the modes $\ell$ such that $\ell \leq m_{\max }$ with $m_{\max }=\left[\left(n_{\lambda_{s}} \kappa_{s} R\right) / 2\right]([x]$ denotes the integer part of a real number $x)$. In practice, the number of modes $m_{\max }$ to retain must be large enough to capture not only the hyperbolic and transition parts of the spectrum but also the elliptic part $\left(m_{\max } \geq \kappa_{s}\right)$, while avoiding the divergence of the spherical Bessel and Hankel functions. The truncation of the series is related to the density $n_{\lambda_{s}}$ of discretization points per S-wavelength $\left(\lambda_{s}=2 \pi / \kappa_{s}\right)$. Keeping more evanescent modes corresponds to the use of a higher density of discretization points in a BEM approximation. 


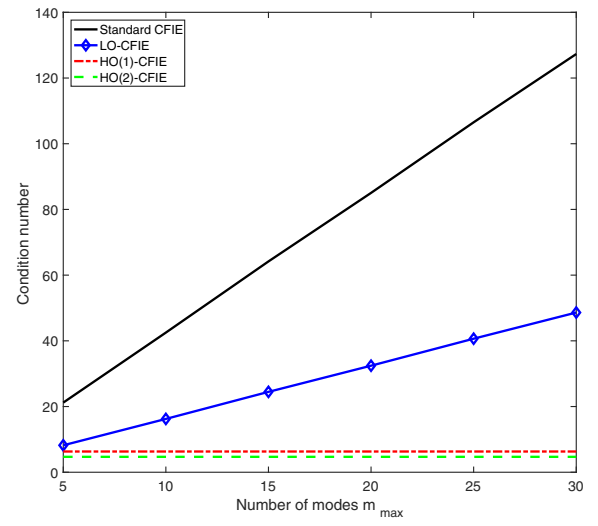

(a)

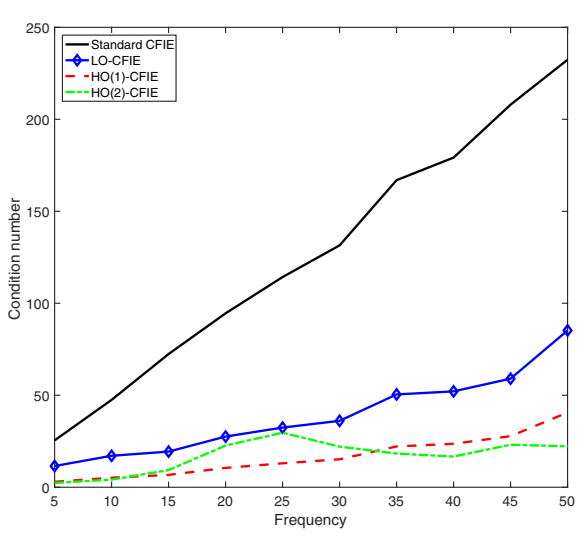

(b)

Fig. 2 Unit sphere. Study of the condition number of the three P-CFIEs. a Condition number with respect to the maximal number of modes $m_{\max }\left(\kappa_{s}=4 \pi\right)$. b Condition number with respect to the frequency $\omega$ $\left(n_{\lambda_{s}}=12\right)$

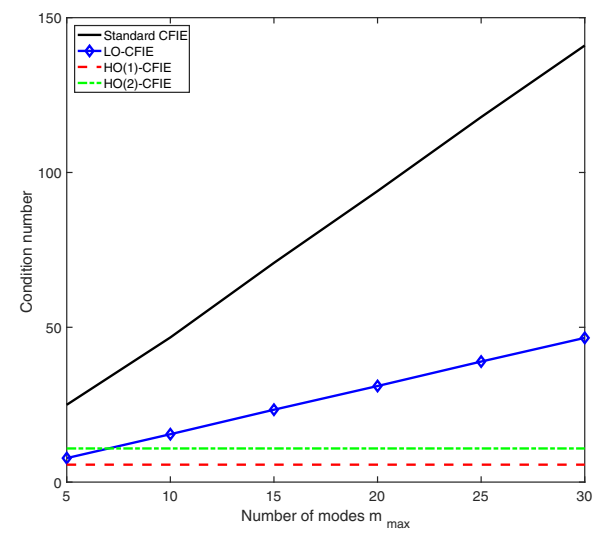

(a)

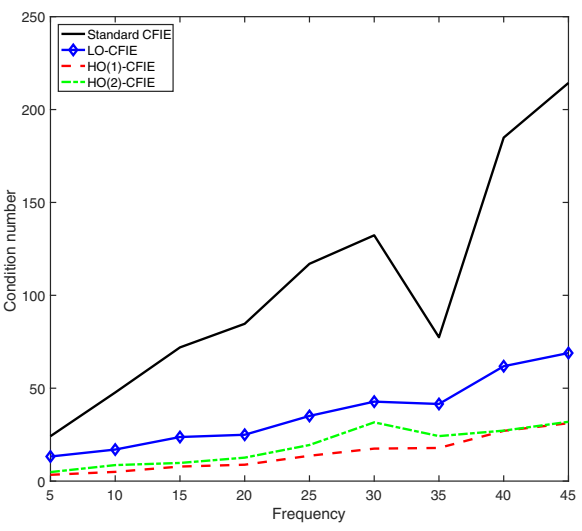

(b)

Fig. 3 Unit disk. Study of the condition number of the P-CFIEs. a Condition number with respect to the maximal number of modes $m_{\max }\left(\kappa_{s}=4 \pi\right)$. b Condition number with respect to the frequency $\left(n_{\lambda_{s}}=12\right)$

In this section, the physical parameters $(\rho=\mu=1, \lambda=3)$ are fixed such that the wavenumbers satisfy $\kappa_{s}=\sqrt{5} \kappa_{p}$. In Fig. 1, we report the eigenvalue distribution of the standard and the proposed P-CFIEs. We observe that the three analytical preconditioners successfully improve the spectral properties of the standard CFIE with an efficient clustering of the eigenvalues. The best results are given by the HO P-CFIEs, particularly in the elliptic zone. As expected, the corresponding condition numbers are independent on the number of modes, i.e., on the mesh density (see Fig. 2), on the contrary to the standard CFIE of the first-kind. Furthermore, the LO and HO P-CFIEs allow to reduce the dependence of the condition number with respect to the frequency. This dependence is linked to the eigenvalues associated with grazing modes. We have also performed this analytical study for the unit disk in 2D and obtain similar conclusions (see Fig. 3). 


\section{Numerical efficiency of analytical preconditioners}

\subsection{Discretization and implementation}

To discretize the surface $\Gamma$ of the scatterer, we consider a triangulation with $N_{E}$ surface triangles and $N_{I}$ vertices. The polyedric interpolated surface is denoted by $\Gamma_{h}$. The discretization is performed by means of classical $\mathbb{P}_{1}$ boundary finite elements. We set $X_{h}=$ $\mathbb{P}_{1}\left(\Gamma_{h}\right)$ and $\boldsymbol{X}_{h}=X_{h}^{d}, d=2,3$, with $\operatorname{dim} \boldsymbol{X}_{h}=M:=d N_{I}$. The application of the $\operatorname{HO}(2)$ preconditioner (20), namely

$$
\boldsymbol{V}_{\mathrm{HO}(2)}^{\prime}=-\left(\frac{\mathrm{I}}{2}-P_{\varepsilon}(D)\right)^{-1} P_{\varepsilon}(S)
$$

is decomposed into the following two steps:

Step 1: Knowing $\boldsymbol{v} \in \boldsymbol{H}^{-\frac{1}{2}}(\Gamma)$, compute the intermediate variable $\boldsymbol{q} \in \boldsymbol{H}^{\frac{1}{2}}(\Gamma)$ such that

$$
\boldsymbol{q}=-P_{\varepsilon}(S) \boldsymbol{v}
$$

Step 2: Knowing $\boldsymbol{q} \in \boldsymbol{H}^{\frac{1}{2}}(\Gamma)$, solve the boundary differential equation: find $\boldsymbol{u} \in \boldsymbol{H}^{\frac{1}{2}}(\Gamma)$ solution to

$$
\left(\frac{\mathrm{I}}{2}-P_{\varepsilon}(D)\right) \boldsymbol{u}=\boldsymbol{q}
$$

The application of the $\mathrm{HO}(1)$-preconditioner (19) reduces to the evaluation of $\boldsymbol{u}=$ $-2 P_{\varepsilon}(S) \boldsymbol{v}$ without the need of the inversion step (Step 2). We refer to [21] for the derivation of the corresponding variational formulation. The efficiency of the HO-preconditioners relies on a robust local representation of the square-root operators $(1+z)^{1 / 2}$, $z \in \mathbb{C}$, and their inverses. To this end, we apply complex Padé approximants with a rotating branch-cut technique, i.e., with a rotation of angle $\theta$ of the usual branch-cut $\{z \in \mathbb{R}, z<1\}$ of the square-root $z \mapsto \sqrt{1+z}$ (see $[22,53]$ ). For $z \in \mathbb{C}$, we have

$$
(1+z)^{1 / 2} \approx e^{i \theta / 2} R_{N_{p}}\left(e^{-i \theta}(1+z)-1\right)=F_{0}-\sum_{\ell=1}^{N_{p}} \frac{A_{\ell}}{B_{\ell}\left(1+B_{\ell} z\right)}
$$

where $R_{N_{p}}$ denotes the standard real-valued Padé approximation and is given by

$$
R_{N_{p}}(z)=1+\sum_{\ell=1}^{N_{p}} \frac{\frac{2}{2 N_{p}+1} \sin ^{2}\left(\ell \pi /\left(2 N_{p}+1\right)\right) z}{1+\cos ^{2}\left(\ell \pi /\left(2 N_{p}+1\right)\right) z} .
$$

The complex constants are then expressed by

$$
A_{\ell}=\frac{e^{-i \theta / 2} \frac{2}{2 N_{p}+1} \sin ^{2}(\ell \pi /(2 L+1))}{\left[1+\cos ^{2}\left(\ell \pi /\left(2 N_{p}+1\right)\right)\left(e^{-i \theta}-1\right)\right]^{2}}, \quad B_{\ell}=\frac{e^{-i \theta} \cos ^{2}\left(\ell \pi /\left(2 N_{p}+1\right)\right)}{\left[1+\cos ^{2}\left(\ell \pi /\left(2 N_{p}+1\right)\right)\left(e^{-i \theta}-1\right)\right]}
$$




$$
F_{0}=e^{i \theta / 2} R_{N_{p}}\left(e^{-i \theta}-1\right)+\sum_{\ell=1}^{N_{p}} \frac{A_{\ell}}{B_{\ell}}
$$

The efficiency of these Padé complex approximants for the local representation of $(1+$ $z)^{1 / 2}$ has been shown numerically in many previous works (e.g., [4, 21, 31]). By choosing a branch cut in the negative half-space, all the modes are modeled correctly and in particular the evanescent ones. In practice, to evaluate the square-root of an operator, we have to solve $N_{p}$ uncoupled sparse linear systems. This step is thus very cheap. We refer to [22] which gives a similar approach for the approximation of the inverse of $\sqrt{1+z}, z \in \mathbb{C}$. The discrete wavenumbers are then expressed by $\kappa_{\gamma, \varepsilon_{h}}=\kappa_{s}+i \varepsilon_{h}$ with $\varepsilon_{h}=0.39 \kappa_{\gamma}^{1 / 3}\left(\mathcal{H}_{h}^{2}\right)^{1 / 3}$. The quantity $\mathcal{H}_{h}$ is a piecewise constant interpolation of the mean curvature $\mathcal{H}$ over $\Gamma_{h}$ on each triangle of the triangulation. The numerical evaluation of $\mathcal{H}_{h}$ comes from the relation

$$
\mathcal{H}_{h}(\boldsymbol{x})=\frac{1}{2} \operatorname{div}_{\Gamma_{h}} \boldsymbol{n}_{h}(\boldsymbol{x})=\frac{1}{2} \sum_{k=1}^{d} \sum_{j=1}^{N_{I}}\left(\boldsymbol{n}_{h}\left(\boldsymbol{a}_{j}\right) \cdot \boldsymbol{e}_{k}\right)\left(\boldsymbol{e}_{k} \cdot \nabla_{\Gamma_{h}} \varphi_{j}(\boldsymbol{x})\right), \quad \boldsymbol{x} \in \Gamma_{h},
$$

where $\boldsymbol{a}_{j}, 1 \leq j \leq N_{I}$, are the vertices of the mesh and the functions $\varphi_{j}, 1 \leq j \leq N_{I}$, the $\mathbb{P}_{1}$ basis functions on $\Gamma_{h}$.

We solve the dense non-symmetric linear systems, corresponding to the BEM discretization of the preconditioned or standard CFIEs, with GMRES [61]. We do not use a restarted version in order to have a precise idea of the impact of the preconditioning technique on the convergence. $[A] \in \mathbb{C}^{M \times M}$ denotes the matrix associated with the linear discretization of a given integral operator $A$. At each iteration of the solver, the solution of the preconditioned CFIE (13) requires the evaluation of the vector $\mathbf{Y} \in \mathbb{C}^{M}$

$$
\mathbf{Y}=\left(\frac{[\mathbf{I}]}{2}-[D]+\left[\boldsymbol{V}^{\prime}\right][N]\right) \mathbf{X}
$$

for any vector $\mathbf{X} \in \mathbb{C}^{M}$ given by GMRES. From a computational point of view, the preconditioners (19) and (20) involve only sparse matrices. Importantly, for the preconditioner (20), Step 2 needs the inversion of $\left.\left(\mathrm{I} / 2-P_{\varepsilon}(D)\right)\right)$. In practice, due to the use of Padé approximations, the matrix associated with this operator is never explicitly assembled and the sparse system is solved with an inner GMRES solver.

To check the efficiency of the proposed analytical preconditioners, we consider three geometries with increasing difficulties. The first geometry is a unit disk used to validate our code. The second one is a unit square with corner singularities. Finally, we construct a Cshape modeling a crack with a finite thickness (see Fig. 4). It is parametrised by $\mathcal{C}=$ $\left\{\left(1.5 \sin \frac{(3 t+4) \pi}{8}-1,0.8 \sin \frac{(3 t+4) \pi}{4}\right):-1 \leq t \leq 1\right\} \cup\left\{\left(1.45 \sin \frac{(3 t+4) \pi}{8}-1,0.7 \sin \frac{(3 t+4) \pi}{4}\right):\right.$

$-1 \leq t \leq 1\}$. For all the examples, we fix the mechanical parameters to $\rho=\mu=1$ and $\lambda=2$ such that $\kappa_{s}=\omega$ and $\kappa_{p}=\omega / 2$. The obstacles are meshed with a density of $n_{\lambda_{s}}$ points per S-wavelength.

\subsection{Spectral analysis}

In order to analyse the convergence properties of GMRES to solve the preconditioned and standard CFIEs, we observe first the eigenvalue behavior of the involved integral operators. The spectral study has been done analytically for the unit disk in Sect. 4. For the unit 
Fig. 4 C-shape: definition of the geometry

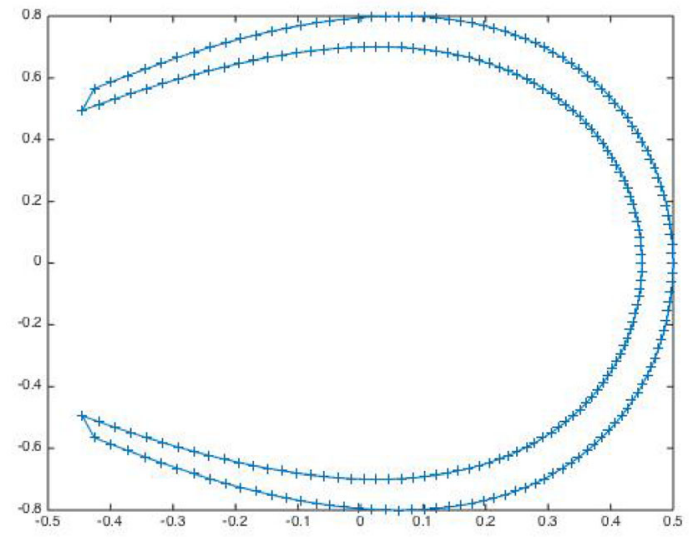

square and the C-shape, an analytical expression of the eigenvalues is not available. We compute them numerically to check if these good spectral properties are also observed. The circular frequency is set to $\omega=2 \pi$, the discretization to $n_{\lambda_{s}}=20$ and the Padé parameter to $N_{p}=60$. We report on Figs. 5 and 6 the eigenvalue distribution for the standard and preconditioned CFIEs when the scatterers are the unit square and the C-shape respectively. For these scatterers, the high-order local approximations of the $\mathrm{NtD}$ map have the remarkable property of clustering the eigenvalues associated with the evanescent modes. This is not the case for the non-preconditioned CFIE. The best spectral behavior is provided by the $\mathrm{HO}(2)$-preconditioner and is favorable for an iterative solution.

\subsection{Numerical efficiency in the context of an iterative solver}

We now compare the convergence of the iterative solver GMRES for the different CFIEs. The scatterers are illuminated by incident plane waves of the form

$$
\boldsymbol{u}^{\mathrm{inc}}(\boldsymbol{x})=\frac{1}{\mu} e^{i \kappa_{s} \boldsymbol{x} \cdot \boldsymbol{d}}(\boldsymbol{d} \times \boldsymbol{p}) \times \boldsymbol{d}+\frac{1}{\lambda+2 \mu} e^{i \kappa_{p} \boldsymbol{x} \cdot \boldsymbol{d}}(\boldsymbol{d} \cdot \boldsymbol{p}) \boldsymbol{d}, \text { where } \boldsymbol{d} \in \mathbf{D}^{2} \text { and } \boldsymbol{p} \in \mathbb{R}^{2}
$$

where $\mathbf{D}^{2}$ is the unit disk in $\mathbb{R}^{2}$. When $\boldsymbol{p}= \pm \boldsymbol{d}$, the incident plane wave oscillates along the direction of propagation (pressure wave or P-wave). When the polarization $\boldsymbol{p}$ is orthogonal to the propagation vector $\boldsymbol{d}$, the incident plane wave oscillates in a direction orthogonal to the direction of propagation (shear wave or S-wave). We consider the scattering of incident plane P-waves with $\boldsymbol{p}=\boldsymbol{d}=(1,0)^{T}$, or $\mathrm{S}$-waves with $\boldsymbol{p}=(1,0)^{T}$ and $\boldsymbol{d}=(0,1)^{T}$. For all the tests, the tolerances of the inner and outer GMRES solvers are set to $10^{-5}$ and $10^{-3}$ respectively. The mechanical parameters are defined such that the wavenumbers satisfy $\kappa_{s}=2 \kappa_{p}$ and $\omega=\kappa_{s}$ (i.e. $\rho=1, \mu=1$ and $\lambda=2$ ) and the number of Padé terms is set to $N_{p}=60$. For the standard CFIE (7), we set the coupling parameter $\eta$ to 1 . But importantly, any constant leads to a similar number of iterations. The conditioning, and hence the number of GMRES iterations, are not very sensitive to this value.

Unit disk. As a usual validation test, we first consider the unit disk. Figure 7 represents the analytical and numerical far fields for $n_{\lambda_{s}}=40$ and $\omega=2 \pi$ and illustrates the accuracy of the code (for incident plane S-waves). The number of GMRES iterations with respect to the frequency $\omega$ are reported in Table 1 (resp. Table 2) for P-waves (resp. S-waves) for the 


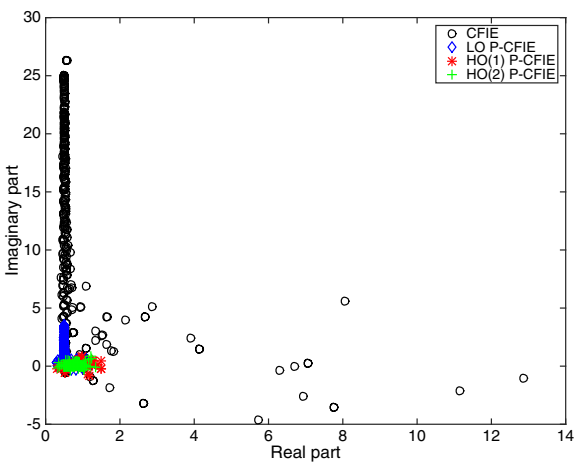

(a)

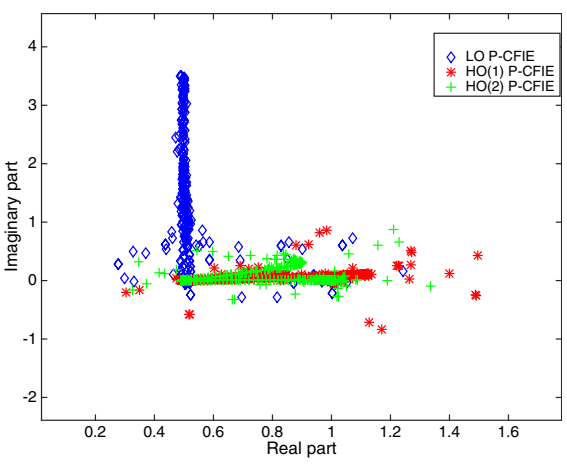

(b)

Fig. 5 Unit square. Distribution of the eigenvalues of the standard and different P-CFIEs $(\eta=1, \omega=2 \pi$ and $n_{\lambda_{s}}=20$ ). a Complete distribution and $\mathbf{b}$ closer view of the clustering

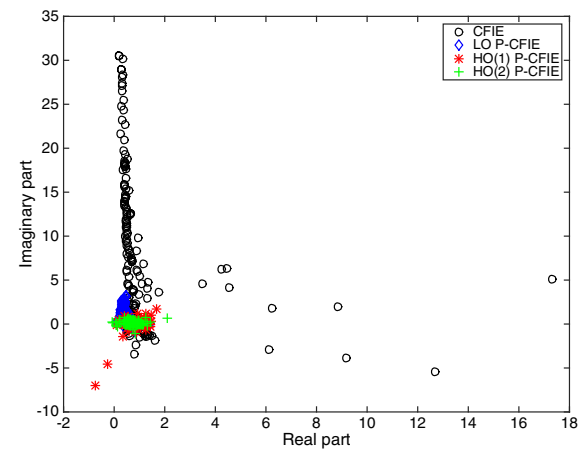

(a)

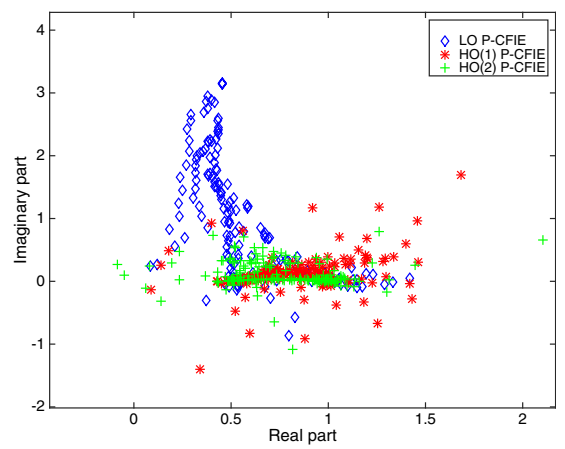

(b)

Fig. 6 C-shape. Distribution of the eigenvalues of the standard and different P-CFIEs $(\eta=1, \omega=2 \pi$ and $n_{\lambda_{s}}=20$ ). a Complete distribution and $\mathbf{b}$ closer view of the clustering

four CFIEs. In the case of the $\mathrm{HO}(2) \mathrm{P}-\mathrm{CFIE}$, inner iterations are indicated in parentheses. The use of the different analytical preconditioners efficiently speeds up the convergence of the solver, particularly at high frequencies. The $\mathrm{HO}(2) \mathrm{P}$-CFIE provides the best results. Nevertheless, the two other P-CFIEs also offer a very good alternative to the standard CFIE and have the advantage to be very simple to implement. The dependence of the convergence with respect to the frequency is linked to eigenvalues corresponding with grazing modes and probably surface modes (Rayleigh waves). In Table 3, we study the number of GMRES iterations with respect to the density of points $n_{\lambda_{s}}$ for incident $\mathrm{S}$-waves. As predicted by the spectral analysis, the convergence is independent on the mesh refinement for the HO P-CFIEs. The LO P-CFIE is also robust with a slight increase of the number of iterations. This is not the case of the standard CFIE which is of the first-kind.

Unit square. We now consider the case of a unit square. The number of GMRES iterations with respect to the frequency $\omega$ is reported in Table 4 (resp. Table 5) for P-waves (resp. S-waves) for the different CFIEs. In Table 6, we study the number of GMRES iterations with respect to the density of points $n_{\lambda_{s}}$ for incident $\mathrm{S}$-waves. For this more 


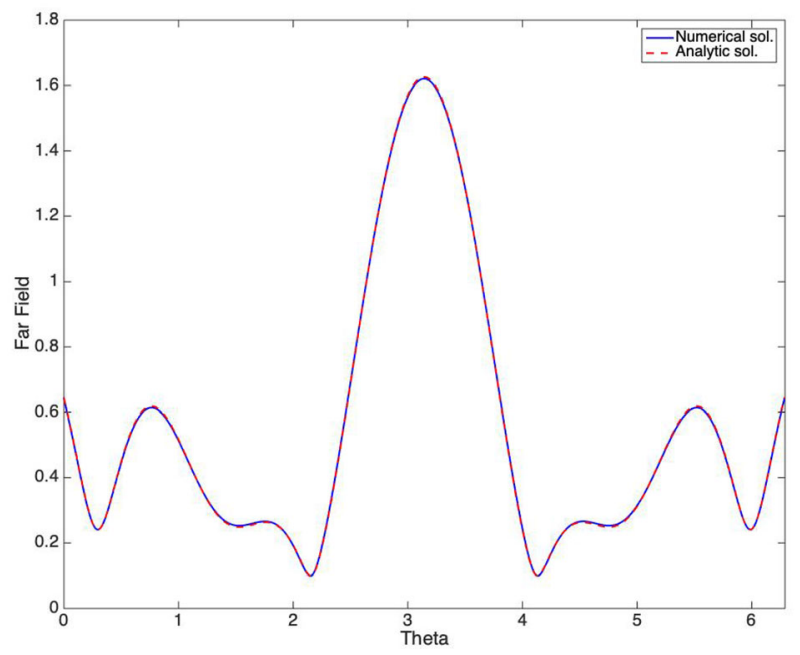

Fig. 7 Unit disk. Validation of the code by comparing the analytical and numerical modulus of the far-field for an incident plane $S$-wave $\left(n_{\lambda_{s}}=40\right.$ and $\left.\omega=2 \pi\right)$. The $L^{2}$-error for this example is $5.8710^{-3}$

Table 1 Unit disk: diffraction of incident P-waves. Number of GMRES iterations for a fixed density of points $n_{\lambda_{s}}=20$

\begin{tabular}{lcccl}
\hline$\omega$ & \# iter CFIE & \# iter LO P-CFIE & \# iter HO(1) P-CFIE & \# iter HO(2) P-CFIE \\
\hline $2 \pi$ & 20 & 6 & 8 & $5(22)$ \\
$4 \pi$ & 32 & 9 & 11 & $4(30)$ \\
$6 \pi$ & 50 & 12 & 11 & $4(32)$ \\
$8 \pi$ & 72 & 13 & 12 & $3(33)$ \\
$16 \pi$ & 120 & 11 & 8 & $3(40)$ \\
$20 \pi$ & 170 & 10 & 8 & $3(45)$ \\
\hline
\end{tabular}

Table 2 Unit disk: diffraction of incident S-waves. Number of GMRES iterations for a fixed density of points $n_{\lambda_{s}}=20$

\begin{tabular}{lllll}
\hline$\omega$ & \# iter CFIE & \# iter LO P-CFIE & \# iter HO(1) P-CFIE & \# iter HO(2) P-CFIE \\
\hline $2 \pi$ & 26 & 13 & 11 & $8(24)$ \\
$4 \pi$ & 46 & 19 & 19 & $8(32)$ \\
$6 \pi$ & 65 & 27 & 25 & $9(39)$ \\
$8 \pi$ & 91 & 36 & 31 & $10(45)$ \\
$16 \pi$ & 186 & 66 & 51 & $14(68)$ \\
\hline
\end{tabular}


Table 3 Unit disk. Diffraction of incident S-waves. Number of GMRES iterations for a fixed frequency $\omega=4 \pi$

\begin{tabular}{lllll}
\hline$n_{\lambda_{s}}$ & \# iter CFIE & \# iter LO P-CFIE & \# iter HO(1) P-CFIE & \# iter HO(2) P-CFIE \\
\hline 5 & 31 & 14 & 19 & $9(30)$ \\
10 & 37 & 16 & 17 & $6(33)$ \\
20 & 46 & 19 & 19 & $8(32)$ \\
30 & 57 & 22 & 19 & $8(32)$ \\
\hline
\end{tabular}

Table 4 Unit square. Diffraction of incident P-waves. Number of GMRES iterations for a fixed density of points $n_{\lambda_{s}}=20$

\begin{tabular}{lllll}
\hline$\omega$ & \# iter CFIE & \# iter LO P-CFIE & \# iter HO(1) P-CFIE & \# iter HO(2) P-CFIE \\
\hline $2 \pi$ & 57 & 19 & 11 & $10(25)$ \\
$4 \pi$ & 103 & 25 & 15 & $11(35)$ \\
$6 \pi$ & 134 & 30 & 21 & $13(42)$ \\
$8 \pi$ & 177 & 36 & 28 & $15(52)$ \\
$16 \pi$ & 287 & 55 & 49 & $20(80)$ \\
\hline
\end{tabular}

Table 5 Unit square. Diffraction of incident S-waves. Number of GMRES iterations for a fixed density of points $n_{\lambda_{s}}=20$

\begin{tabular}{lllll}
\hline$\omega$ & \# iter CFIE & \# iter LO P-CFIE & \# iter HO(1) P-CFIE & \# iter HO(2) P-CFIE \\
\hline $2 \pi$ & 63 & 21 & 12 & $10(25)$ \\
$4 \pi$ & 120 & 26 & 18 & $12(35)$ \\
$6 \pi$ & 171 & 32 & 23 & $15(43)$ \\
$8 \pi$ & 226 & 38 & 29 & $17(52)$ \\
$16 \pi$ & 421 & 70 & 53 & $24(80)$ \\
\hline
\end{tabular}

difficult geometry, the number of iterations without any preconditioner substantially increases with both the frequency and the mesh refinement. The three preconditioners are efficient. Here again, the dependence on the frequency after preconditioning is reduced, and the independence of the convergence according to a mesh refinement is still observed with the HO P-CFIEs.

$C$-shape. It is known that the dependence on the frequency is more pronounced for the case of a trapping obstacle than for the case of a disk or square. It is interesting to study the efficiency of analytical preconditioners for such scattering objects. The number of GMRES iterations with respect to the frequency $\omega$ is reported in Table 7 (resp. Table 8) for P-waves (resp. S-waves) for the four CFIEs. The number of iterations without any preconditioner drastically increases with the frequency. The three preconditioners are seen to be very 
Table 6 Unit square. Diffraction of incident S-waves. Number of GMRES iterations for a fixed frequency $\omega=16 \pi$

\begin{tabular}{lllll}
\hline$n_{\lambda_{s}}$ & \# iter CFIE & \# iter LO P-CFIE & \# iter HO(1) P-CFIE & \# iter HO(2) P-CFIE \\
\hline 20 & 421 & 70 & 53 & $24(80)$ \\
30 & 449 & 86 & 55 & $23(80)$ \\
40 & 475 & 99 & 55 & $24(80)$ \\
\hline
\end{tabular}

Table 7 C-shape: diffraction of incident P-wave. Number of GMRES iterations for a fixed density of points $n_{\lambda_{s}}=20$

\begin{tabular}{lllll}
\hline$\omega$ & \# iter CFIE & \# iter LO P-CFIE & \# iter HO(1) P-CFIE & \# iter HO(2) P-CFIE \\
\hline $2 \pi$ & 66 & 27 & 25 & $23(40)$ \\
$4 \pi$ & 106 & 34 & 31 & $45(54)$ \\
$6 \pi$ & 176 & 47 & 45 & $52(73)$ \\
$8 \pi$ & 207 & 44 & 41 & $71(84)$ \\
$16 \pi$ & 332 & 61 & 52 & $99(141)$ \\
\hline
\end{tabular}

Table 8 C-shape: Diffraction of incident S-wave. Number of GMRES iterations for a fixed density of points $n_{\lambda_{s}}=20$

\begin{tabular}{lrllll}
\hline$\omega$ & N & \# iter CFIE & \# iter LO P-CFIE & \# iter HO(1) P-CFIE & \# iter HO(2) P-CFIE \\
\hline $2 \pi$ & 81 & 65 & 26 & 25 & $24(40)$ \\
$4 \pi$ & 165 & 122 & 35 & 31 & $45(54)$ \\
$6 \pi$ & 250 & 180 & 47 & 46 & $55(72)$ \\
$8 \pi$ & 331 & 205 & 51 & 41 & $71(83)$ \\
$16 \pi$ & 666 & 328 & 69 & 56 & $104(140)$ \\
$20 \pi$ & 829 & 395 & 76 & 61 & $114(173)$ \\
\hline
\end{tabular}

efficient (with at least the number of iterations divided by three). The dependence on the frequency after preconditioning is reduced. Importantly, for this geometry HO(1) P-CFIE is the most robust approach particularly at high frequencies. For example, where multiplying the frequency by 8 , the number of iterations is only multiplied by a factor 2 . The relative loss of performance of the $\mathrm{HO}(2)$ P-CFIE approach for this geometry can be explained by the inversion of the operator $\left(\frac{\mathrm{I}}{2}-P_{\varepsilon}(D)\right)$ in $(25)$, where $P_{\varepsilon}(D)$ is the principal part of the non-compact operator $D$. For Dirichlet problems, we proposed to consider a modified potential theory (using the tangential Günter derivative) in which the corresponding double-layer boundary integral operator is a compact operator [31, 32]. This technique is not possible for Neumann problems. The inversion in (25) requires a higher 
Padé order to get a good approximation of the adjoint NtD and to make the $\mathrm{HO}$ (2) P-CFIE competitive. The $\mathrm{HO}(1) \mathrm{P}-\mathrm{CFIE}$ is a very interesting compromise for such configurations.

All these numerical examples show the efficiency of the three proposed P-CFIEs. To determine which preconditioner should be used in practice, it is not only important to consider the number of iterations to achieve convergence but also the computational time added to evaluate the preconditioner. Obviously, the LO P-CFIE does not add any cost. On the other hand, the HO P-CFIEs require the inversion of some sparse linear systems. These systems are embarrassingly parallel so that, with very little implementation effort, the additional computational cost is small. However, the HO(1) P-CFIE is simpler and has been shown to be very efficient including for complex geometries. For Neumann scattering problems, it is currently the most efficient approach from the authors' point of view. In order to improve the numerical approximation of the inversion of $\left(\frac{\mathrm{I}}{2}-P_{\varepsilon}(D)\right)$ in $(25)$ and hence the robustness of the $\mathrm{HO}(2) \mathrm{P}-\mathrm{CFIE}$ for any scatterer, a perspective is to propose another method for the local representation of the square-root operator and its inverse than the use of complex Padé approximants.

\section{Conclusion and future work}

In this paper, we have presented a first conclusive study of the efficiency of analytical preconditioners for the numerical solution of high-oscillatory Neumann elastic exterior problems. We have proposed three preconditioners corresponding to approximations with various orders of the exact adjoint Neumann-to-Dirichlet (NtD) map. The theory based on the calculus of the principal pseudo-differential symbol of the NtD map is described in two- and three-dimensions.

A spectral analytical study for the spherical case has shown that the high-order preconditioned CFIEs are not of the first-kind whereas non-preconditioned CFIEs are. Consequently, their condition numbers are not sensitive to the mesh refinement and the convergence of GMRES is independent of the mesh size too. Numerical simulations on various $2 \mathrm{D}$ geometries have attested that the convergence of GMRES is also greatly improved with respect to the frequency with the use of high-order and local approximations of the NtD. A slight dependence on the frequency is observed. Nevertheless, the proposed preconditioned CFIEs offer a very competitive alternative to the standard one.

Ongoing work concerns a more extensive analysis to understand the influence in the preconditioner of some physical aspects inherent to a cavity problem, e.g., surface Rayleigh waves.

\section{References}

1. Alouges, F., Borel, S., Levadoux, D.P.: A stable well-conditioned integral equation for electromagnetism scattering. J. Comput. Appl. Math. 204, 440-451 (2007)

2. Antoine, X., Barucq, H., Bendali, A.: Bayliss-turkel-like radiation condition on surfaces of arbitrary shape. J. Math. Anal. Appl. 229, 184-211 (1999)

3. Antoine, X., Bendali, A., Darbas, M.: Analytic preconditioners for the boundary integral solution of the scattering of acoustic waves by open surfaces. J. Comput. Acoust. 13, 477-498 (2005)

4. Antoine, X., Darbas, M.: Alternative integral equations for the iterative solution of acoustic scattering problems. Quart. J. Mech. Appl. Math. 58, 107-128 (2005)

5. Antoine, X., Darbas, M.: Generalized combined field integral equations for the iterative solution of the three-dimensional Helmholtz equation, M2AN Math. Model. Numer. Anal. 41, 147-167 (2007) 
6. Antoine, X., Darbas, M., Lu, Y.Y.: An improved surface radiation condition for high-frequency acoustic scattering problems. Comput. Methods Appl. Mech. Engrg. 195, 4060-4074 (2006)

7. Bebendorf, M., Grzhibovskis, R.: Accelerating galerkin bem for linear elasticity using adaptive cross approximation. Math. Methods Appl. Sci. 29, 1721-1747 (2006)

8. Berenger, J.P.: A perfectly matched layer for the absorption of electromagnetic waves. J. Comput. Phys. 114, 185-200 (1994)

9. Bielak, J., Loukakis, K., Hisada, Y., Yoshimura, C.: Domain reduction method for three-dimensional earthquake modeling in localized regions, part i: Theory. Bull. Seismol. Soc. Am. 93, 817-824 (2003)

10. Bonnet, M.: Boundary Integral Equations Methods in Solids and Fluids. Wiley, Amsterdam (1999)

11. Boubendir, Y., Turc, C.: Well-conditioned boundary integral equation formulations for the solution of high-frequency electromagnetic scattering problems. Comput. Math. Appl. 67, 1772-1805 (2014)

12. Bruno, O., Elling, T., Paffenroth, R., Turc, C.: Electromagnetic integral equations requiring small numbers of krylov-subspace iterations. J. Comput. Phys. 228, 6169-6183 (2009)

13. Bruno, O., Elling, T., Turc, C.: Regularized integral equations and fast high-order solvers for soundhard acoustic scattering problems. Int. J. Numer. Meth. Eng. 91, 1045-1072 (2012)

14. Bruno, O., Lintner, S.: Second-kind integral solvers for TE and TM problems of diffraction by open arcs. Radio Sci. 47 (2012)

15. Carpentieri, B.: A matrix-free two-grid preconditioner for solving boundary integral equations in electromagnetism. Computing 77, 275-296 (2006)

16. Carpentieri, B., Duff, I., Giraud, L.: Some sparse pattern selection strategies for robust frobenius norm minimization preconditioners in electromagnetism. Numer. Linear Algebra Appl. 7, 667-685 (2000)

17. Carpentieri, B., Duff, I.S., Giraud, L., Sylvand, G.: Combining fast multipole techniques and an approximate inverse preconditioner for large parallel electromagnetics calculations. SIAM J. Sci. Comp. 27, 774-792 (2005)

18. Cerjan, C., Kosloff, D., Kosloff, R., Reshef, M.: A nonreflecting boundary condition for discrete acoustic and elastic wave equations. Geophysics 50, 705-708 (1985)

19. Chaillat, S., Bonnet, M.: Recent advances on the fast multipole accelerated boundary element method for 3D time-harmonic elastodynamics. Wave Motion 50, 1090-1104 (2013)

20. Chaillat, S., Bonnet, M., Semblat, J.F.: A multi-level fast multipole BEM for 3-D elastodynamics in the frequency domain. Comput. Methods Appl. Mech. Eng. 197, 4233-4249 (2008)

21. Chaillat, S., Darbas, M., Le Louër, F.: Approximate local dirichlet-to-neumann map for three-dimensional time-harmonic elastic waves. Comput. Methods Appl. Mech. Eng. 297, 62-83 (2015)

22. Chaillat, S., Darbas, M., Le Louër, F.: Fast iterative boundary element methods for high-frequency scattering problems in 3d elastodynamics. J. Comput. Phys. 341, 429-446 (2017)

23. Chaillat, S., Semblat, J.F., Bonnet, M.: A preconditioned 3-d multi-region fast multipole solver for seismic wave propagation in complex geometries. Commun. Comput. Phys. 11, 594-609 (2012)

24. Chaljub, E., Komatitsch, D., Vilotte, J.-P., Capdeville, Y., Valette, B., Festa, G.: Spectral-element analysis in seismology. Adv. Geophys. 48, 365-419 (2007)

25. Chandler-Wilde, S., Graham, I.G., Langdon, S., Spence, E.A.: Numerical-asymptotic boundary integral methods in high-frequency acoustic scattering. Acta Numer. 21, 89-305 (2012)

26. Chew, W.C., Liu, Q.H.: Perfectly matched layers for elastodynamics: a new absorbing boundary condition. J. Comput. Acoust. 4, 341-359 (1996)

27. Christiansen, S.: Discrete fredholm properties and convergence estimates for the electric field integral equation. Math. Comp. 73, 143-167 (2004)

28. Christiansen, S., Nédélec, J.C.: A preconditioner for the electric field integral equation based on calderon formulas. SIAM J. Numer. Anal. 40, 1100-1135 (2002)

29. Clayton, R., Engquist, B.: Absorbing boundary conditions for acoustic and elastic wave equations. Bull. Seismol. Soc. Am. 67, 1529-1540 (1977)

30. Darbas, M.: Generalized combined field integral equations for the iterative solution of the threedimensional Maxwell equations. Appl. Math. Lett. 19, 834-839 (2006)

31. Darbas, M., Darrigrand, E., Lafranche, Y.: Combining OSRC preconditioning and Fast Multipole Method for the Helmholtz equation. J. Comput. Phys. 236, 289-316 (2013)

32. Darbas, M., Le Louër, F.: Well-conditioned boundary integral formulations for high-frequency elastic scattering problems in three dimensions. Math. Methods Appl. Sci. 38, 1705-1733 (2015)

33. El Bouajaji, M., Antoine, X., Geuzaine, C.: Approximate local magnetic-to-electric surface operators for time-harmonic maxwell's equations. J. Comput. Phys. 279, 241-260 (2014)

34. Engquist, B., Majda, A.: Radiation boundary conditions for acoustic and elastic wave calculations. Commun. Pure Appl. Math. 32, 314-358 (1979)

35. Escapil-Inchauspé, P., Jerez-Hanckes, C.: Fast calderón preconditioning for the electric field integral equation. IEEE Trans. Antennas Propag. 67, 2555-2564 (2019) 
36. Fierro, I., Jerez-Hanckes, C.: Fast calderón preconditioning for helmholtz boundary integral equations. J. Comput. Phys., p. 109355 (2020)

37. Gächter, G.K., Grote, M.J.: Dirichlet-to-Neumann map for three-dimensional elastic waves. Wave Motion 37, 293-311 (2003)

38. Givoli, D.: High-order nonreflecting boundary conditions without high-order derivatives. J. Comput. Phys. 122, 849-870 (2001)

39. Graves, R.W.: Simulating seismic wave propagation in 3D elastic media using staggered-grid finite differences. Bull. Seismol. Soc. Am. 86, 1091-1106 (1996)

40. Halpern, L., Petit-Bergez, S., Rauch, J.: The analysis of matched layers. Confluentes Mathematici 3, 159-236 (2011)

41. Hesthaven, J.S., Warburton, T.: Nodal Discontinuous Galerkin Methods: Algorithms, Analysis, and Applications, vol. 54. Springer, New York (2007)

42. Hsiao, G.C., Wendland, W.L.: Boundary Integral Equations. Applied Mathematical Sciences, vol. 164. Springer, Berlin (2008)

43. Jones, D.S.: An approximate boundary condition in acoustics. J. Sound Vib. 121, 37-45 (1998)

44. Komatitsch, D., Vilotte, J.P.: The spectral element method: an efficient tool to simulate the seismic response of 2D and 3D geological structures. Bull. Seismol. Soc. Am. 88, 368-392 (1998)

45. Kpadonou, F., Chaillat, S., Ciarlet Jr., P.: On the efficiency of nested gmres preconditioners for 3D acoustic and elastodynamic $\mathcal{H}$-matrix accelerated boundary element methods. Comput. Math. Appl. 30, 471-489 (2020)

46. Kress, R.: Minimizing the condition number of boundary integral operators in acoustic and electromagnetic scattering. Quart. J. Mech. Appl. Math. 38, 323-341 (1985)

47. Kriegsmann, G.A., Taflove, A., Umashankar, K.R.: A new formulation of electromagnetic wave scattering using the on-surface radiation condition method. IEEE Trans. Antennas Propag. 35, 153-161 (1987)

48. Kupradze, V.D.: Potential methods in the theory of elasticity, Translated from the Russian by H. Gutfreund. Translation edited by I. Meroz, Israel Program for Scientific Translations, Jerusalem (1965)

49. Kupradze, V.D., Gegelia, T.G., Bashelě̌shvili, M., Burchuladze, T.V.: Three-dimensional problems of the mathematical theory of elasticity and thermoelasticity, vol. 25 of North-Holland Series in Applied Mathematics and Mechanics, North-Holland Publishing Co., Amsterdam, russian ed., (1979). Edited by V. D. Kupradze

50. Le Louër, F.: A high-order spectral algorithm for elastic obstacle scattering in three dimensions. J. Comput. Phys. 279, 1705-1733 (2014)

51. Levadoux, D.P.: Proposition de préconditionneurs pseudo-différentiels pour l'équation cfie de l'électromagnétisme., M2AN, 39, pp. 147-155 (2005)

52. Levadoux, D.P., Michielsen, B.L.: Nouvelles formulations intégrales pour les problèmes de diffraction d'ondes. M2AN, 38, 157-175 (2004)

53. Milinazzo, F.A., Zala, C.A., Brooke, G.H.: Rational square-root approximations for parabolic equation algorithms. J. Acoust. Soc. Am. 101, 760-766 (1997)

54. Nédélec, J.-C.: Acoustic and electromagnetic equations, vol. 144 of Applied Mathematical Sciences, Springer-Verlag, New York. Integral representations for harmonic problems (2001)

55. Pernet, S.: A well-conditioned integral equation for iterative solution of scattering problems with a variable Leontovitch boundary condition, M2AN Math. Model. Numer. Anal. 44, 781-801 (2010)

56. Rokhlin, V.: Rapid solution of integral equations of scattering theory in two dimensions. J. Comput. Phys. 86, 414-439 (1990)

57. Saenger, E.H., Gold, N., Shapiro, S.A.: Modeling the propagation of elastic waves using a modified finite-difference grid. Wave Motion 31, 77-92 (2000)

58. Steinbach, O., Wendland, W.L.: The construction of some efficient preconditioners in the boundary element method. Adv. Comput. Math. 9, 191-216 (1998)

59. Stevenson, R., van Venetië, R.: Uniform preconditioners for problems of positive order. Comput. Math. Appl. (2020)

60. Virieux, J., Calandra, H., Plessix, R.E.: A review of the spectral, pseudo-spectral, finite-difference and finite-element modelling techniques for geophysical imaging. Geophys. Prospect. 59, 794-813 (2011)

61. Saad, Y.: Iterative Methods for Sparse Linear Systems. PWS Publishing Company, Boston (1996) 


\section{Affiliations}

\section{Stéphanie Chaillat ${ }^{1} \cdot$ Marion Darbas $^{2} \cdot$ Frédérique Le Louër $^{3}$}

$\bowtie$ Marion Darbas

darbas@math.univ-paris13.fr

Stéphanie Chaillat

stephanie.chaillat@ensta-paris.fr

Frédérique Le Louër

frederique.le-louer@utc.fr

1 Laboratoire POEMS (CNRS-INRIA-ENSTA Paris), Institut Polytechnique de Paris, ENSTAUMA, 828 Bd des Maréchaux, 91762 Palaiseau Cedex, France

2 LAGA UMR CNRS 7539, Institut Galilée, Université Sorbonne Paris Nord, 99 avenue Jean Baptiste Clément, 93430 Villetaneuse, France

3 LMAC EA 2222, Sorbonne Universités, Université de technologie de Compiègne, CS 60319 , 60203 Compiègne cedex, France 